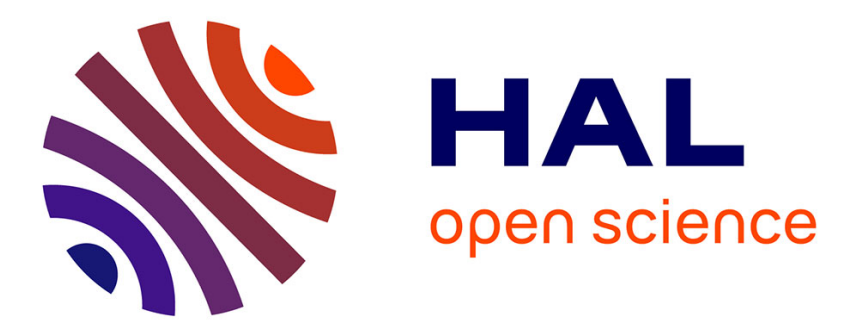

\title{
f-statistics estimation and admixture graph construction with Pool-Seq or allele count data using the $R$ package poolfstat
}

\author{
Mathieu Gautier, Renaud Vitalis, Laurence Flori, Arnaud Estoup
}

\section{To cite this version:}

Mathieu Gautier, Renaud Vitalis, Laurence Flori, Arnaud Estoup. f-statistics estimation and admixture graph construction with Pool-Seq or allele count data using the $\mathrm{R}$ package poolfstat. Molecular Ecology Resources, 2022, 22 (4), pp.1394-1416. 10.1111/1755-0998.13557 . hal-03481066

\section{HAL Id: hal-03481066 \\ https://hal.inrae.fr/hal-03481066}

Submitted on 14 Jan 2022

HAL is a multi-disciplinary open access archive for the deposit and dissemination of scientific research documents, whether they are published or not. The documents may come from teaching and research institutions in France or abroad, or from public or private research centers.
L'archive ouverte pluridisciplinaire HAL, est destinée au dépôt et à la diffusion de documents scientifiques de niveau recherche, publiés ou non, émanant des établissements d'enseignement et de recherche français ou étrangers, des laboratoires publics ou privés.

\section{(1) (1) $\$$}

Distributed under a Creative Commons Attribution - NonCommercial - NoDerivatives| 4.0 


\title{
$f$-statistics estimation and admixture graph construction with Pool-Seq or allele count data using the $\mathrm{R}$ package poolfstat
}

\author{
Mathieu Gautier ${ }^{1, \star}$, Renaud Vitalis ${ }^{1}$, Laurence Flori ${ }^{2}$ and Arnaud Estoup ${ }^{1}$ \\ ${ }^{1}$ CBGP, INRAE, CIRAD, IRD, Montpellier SupAgro, Univ Montpellier, Montpellier, France \\ ${ }^{2}$ SelMet, INRAE, CIRAD, Montpellier SupAgro, Montpellier, France
}

${ }^{\star}$ Corresponding author:

Mathieu Gautier

INRAE-CBGP (Centre de Biologie pour la Gestion des Populations), 755 avenue du campus Agropolis,

CS30016, 34988 Montferrier sur lez cedex

Tel: +33499623331; Fax:+33499623345

E-mail: mathieu.gautier@inrae.fr

Keywords: $f$-statistics; Admixture Graph; Pool-Seq; Demographic Inference; Drosophila suzukii

Running title: Inferring demographic history with the R package poolfstats v2.0 


\section{Abstract}

By capturing various patterns of the structuring of genetic variation across populations, $f$-statistics have proved

3 highly effective for the inference of demographic history. Such statistics are defined as covariance of SNP allele

4 frequency differences among sets of populations without requiring haplotype information and are hence particu-

5 larly relevant for the analysis of pooled sequencing (Pool-Seq) data. We here propose a reinterpretation of the $F$

6 (and $D$ ) parameters in terms of probability of gene identity and derive from this unified definition unbiased estima-

7 tors for both Pool-Seq and standard allele count data obtained from individual genotypes. We implemented these

8 estimators in a new version of the R package poolfstat, which now includes a wide range of inference methods:

9 (i) three-population test of admixture; (ii) four-population test of treeness; (iii) $F_{4}$-ratio estimation of admixture rates; and (iv) fitting, visualization and (semi-automatic) construction of admixture graphs. A comprehensive evaluation of the methods implemented in poolfstat on both simulated Pool-Seq (with various sequencing coverages and error rates) and allele count data confirmed the accuracy of these approaches, even for the most cost-effective Pool-Seq design involving low sequencing coverages. We further analyzed a real Pool-Seq data made of 14 populations of the invasive species Drosophila suzukii which allowed refining both the demographic history of native populations and the invasion routes followed by this emblematic pest. Our new package poolfstat provides the community with a user-friendly and efficient all-in-one tool to unravel complex population genetic histories from 


\section{Introduction}

In their seminal paper, Reich et al (2009) introduced a new population genetics framework to decipher the history of Indian human populations. This inference approach relied on a set of so-called $f$-statistics that are aimed at capturing various patterns of the structuring of genetic diversity across-population based on Single Nucleotide Polymorphism (SNP) assayed on a genome-wide scale (see also Patterson et al, 2012). The parameters underlying these statistics and denoted $F$ following Patterson et al (2012) are defined as covariances in allele frequency difference among sets of two $\left(F_{2}\right)$, three $\left(F_{3}\right)$ or four $\left(F_{4}\right)$ populations and were demonstrated to be highly informative about populations demographic history when modeled as admixture graphs, i.e., population trees possibly including admixture events (Patterson et al, 2012). Hence, formal tests of admixture, called three-population test, between a target population and two source population surrogates can be derived from estimates of $F_{3}$. Conversely, via the so-called four-population test, estimating $F_{4}$ among quadruplets of populations allows to test for their treeness, i.e., if their joint history can be modeled as a simple (unrooted) bifurcating tree. Under certain restrictive assumptions about the underlying phylogeny, accurate estimates of the relative contributions of the ancestral sources of an admixed population may be obtained from ratios of $F_{4}$ involving some of its related sampled populations. A normalized version of the $F_{4}$ parameter, called Patterson's $D$, was also developed by Green $e t$ al (2010) and has become quite popular to characterize introgression in phylogenies of closely related species (Durand et al, 2011). Finally, $f$-statistics can directly be used to fit admixture graphs (i.e., estimate branch lengths and/or admixture proportions) and to rigorously assess their support (Patterson et al, 2012; Lipson et al, 2013; Lipson, 2020).

A critical advantage of $F$ and $D$ parameters is that they only depend on population allele frequencies and their estimation does not require haplotype information. The non-independence of neighboring SNPs (Linkage Disequilibrium or LD) can be accurately accounted for with block-jackknife statistical techniques (Patterson et al, 2012; Kunsch, 1989; Reich et al, 2009; Busing et al, 1999) when computing standard errors of the estimated $f$-statistics which are noticeably required for the derivation of formal tests of admixture or treeness and also to assess the residuals of fitted admixture graphs. These characteristics make the $f$-statistics based inference framework particularly attractive for the analysis of Pool-Seq data that result from the massive sequencing of pools of individual DNA and have become quite popular, most particularly in non-model species (Schlötterer et al, 2014). Indeed, although LD information is generally lost in Pool-Seq experiments (but see Long et al, 2011; or Feder et al, 2012), they lead to accurate and cost-effective assessment of allele frequencies across populations on a 
whole genome basis (Gautier et al, 2013; Schlötterer et al, 2014). If the derivation of unbiased estimates of allele frequencies from Pool-Seq data is straightforward, estimation of more elaborated population genetics parameters characterizing the structuring of genetic diversity within or across populations is more challenging (Gautier et al, 2013; Ferretti et al, 2013; Hivert et al, 2018). As the individual origin of the sequencing reads is not identifiable within pools, it is not possible to assess whether reads are identical because they are sequenced copies of the same individual chromosome or because they are copies of different chromosomes carrying the same allele. The resulting additional level of variation thus needs to be accounted for in the estimation which, in contrast to the nucleotide diversities (heterozygosities) or the well-known $F_{S T}$ differentiation measure (Ferretti et al, 2013; Hivert et al, 2018), has to our knowledge not been investigated for the estimation of $F$ and $D$ parameters (but see Leblois et al, 2018; Collin et al, 2021).

In the present paper, we first propose a (re)interpretation of the different $F$ and $D$ parameters in terms of probability of identity in state (IIS or AIS for Alike-In-State) of pairs of genes sampled either within the same population $\left(Q_{1}\right)$ or between two different populations $\left(Q_{2}\right)$, extending results we introduced in some earlier studies (Hivert et al, 2018; Leblois et al, 2018; Collin et al, 2021). This unified definition simplified the derivation of the unbiased estimators for both allele-count and Pool-Seq read count data, that we implemented in a new version of our R package poolfstat (Hivert et al, 2018) together with methods that rely on the estimated fstatistics for historical and demographic inference. These methods include i) three-population test of admixture; ii) four-population test of treeness; iii) $F_{4}$-ratio estimation of admixture proportion; and iv) fitting, visualization and (semi-automatic) construction of admixture graphs. For completion, we briefly present the underlying methods as implemented in the package. We then carried out a comprehensive evaluation of the whole package on both simulated allele count and Pool-Seq read count data, considering for the latter various sequencing coverages and the presence or not of sequencing errors. Finally, we illustrate the power and limitations of poolfstat by analyzing real Pool-Seq data available from a previous study (Olazcuaga et al, 2020) for 14 populations of the invasive species Drosophila suzukii. This example illustrates how $f$-statistics based inference and admixture graph construction may confirm previous inferences and provide new insights into both the history of populations from the native area and the invasion routes followed by an emblematic invasive species. We provide as Supplementary Materials, a first vignette (Supplementary Vignette V1) designed as a detailed hands-on manual to outline the main functionalities of poolfstat and a second vignette (Supplementary Vignette V2) detailing the analysis of the D. suzukii data to make it fully reproducible. 


\section{Material and Methods}

\subsection{Definition, estimation and $f$-statistics based inference methods}

\subsubsection{A unified definition of $F_{2}, F_{3}$ and $F_{4}$ parameter and their scaled version $F_{S T}, F_{3}^{\star}$ and $D$ in terms of} $Q_{1}$ and $Q_{2}$ probabilities

Let $p_{A}, p_{B}, p_{C}$ and $p_{D}$ the allele frequency of an arbitrarily chosen allele at a random SNP segregating in populations $A, B, C$ and $D$ respectively. The parameters $F_{2}, F_{3}$ and $F_{4}$ were originally defined in terms of covariance in allele frequencies difference among different sets of populations as follows (Reich et al, 2009; Patterson et al, 2012):

- $F_{2}(A ; B) \quad \equiv \mathbb{E}\left[\left(p_{A}-p_{B}\right)^{2}\right]$

- $F_{3}(A ; B, C) \quad \equiv \mathbb{E}\left[\left(p_{A}-p_{B}\right)\left(p_{A}-p_{C}\right)\right]=\frac{1}{2}\left(F_{2}(A ; B)+F_{2}(A ; C)-F_{2}(B ; C)\right)$

- $F_{4}(A, B ; C, D) \equiv \mathbb{E}\left[\left(p_{A}-p_{B}\right)\left(p_{C}-p_{D}\right)\right]=\frac{1}{2}\left(F_{2}(A ; D)+F_{2}(B ; C)-F_{2}(A ; C)-F_{2}(B ; D)\right)$

In total, with $n$ populations, there are $\left(\begin{array}{l}n \\ 2\end{array}\right)=\frac{1}{2} n(n-1)$ possible $F_{2} ; 3\left(\begin{array}{l}n \\ 3\end{array}\right)=\frac{1}{2} n(n-1)(n-2)$ possible $F_{3}$; and $3\left(\begin{array}{l}n \\ 4\end{array}\right)=\frac{1}{8} n(n-1)(n-2)(n-3)$ possible $F_{4}$. Note that these values exclude the alternative equivalent configurations that result from the permutation of populations within pairs (since $F_{2}(A ; B)=F_{2}(B ; A) ; F_{3}(A ; B, C)=F_{3}(A ; C, B)$ and $\left.F_{4}(A, B ; C, D)=F_{4}(B, A ; D, C)=-F_{4}(B, A ; C, D) \ldots\right)$. Due to the linear dependency of all these parameters (eq. 1), the $\frac{1}{8} n(n-1)\left(n^{2}-n+2\right) F$ parameters actually span a vector space of dimension $\frac{1}{2} n(n-1)$ the basis of which may be specified by the set of all the $\left(\begin{array}{l}n \\ 2\end{array}\right)$ possible $F_{2}$ or, given a reference population $i$ (randomly chosen among the $n$ ones) the set of all the $n-1 F_{2}$ of the form $F_{2}(i ; j)$ (with $j \neq i$ ) and all the $\left(\begin{array}{c}n-1 \\ 2\end{array}\right) F_{3}$ of the form $F_{3}(i ; j, k)$ (with $j \neq i ; k \neq i$ and $j \neq k$ ) (Patterson et al, 2012; Lipson, 2020). As mentioned by Patterson $e t$ al (2012), it is important to notice that these definitions are invariant in the choice of the reference SNP allele since:

$$
F_{2}(A ; B) \equiv \mathbb{E}\left[\left(p_{A}-p_{B}\right)^{2}\right]=\mathbb{E}\left[\left(\left(1-p_{A}\right)-\left(1-p_{B}\right)\right)^{2}\right]
$$

It directly follows from this property that: 
94

$$
\begin{aligned}
F_{2}(A ; B) & =\frac{1}{2}\left(\mathbb{E}\left[\left(p_{A}-p_{B}\right)^{2}\right]+\mathbb{E}\left[\left(\left(1-p_{A}\right)-\left(1-p_{B}\right)\right)^{2}\right]\right) \\
& =\frac{1}{2}\left(\mathbb{E}\left[p_{A}^{2}\right]+\mathbb{E}\left[\left(1-p_{A}\right)^{2}\right]\right)+\frac{1}{2}\left(\mathbb{E}\left[p_{B}^{2}\right]+\mathbb{E}\left[\left(1-p_{B}\right)^{2}\right]\right)-\left(\mathbb{E}\left[p_{A} p_{B}\right]+\mathbb{E}\left[\left(1-p_{A}\right)\left(1-p_{B}\right)\right]\right) \\
& =\frac{Q_{1}^{A}+Q_{1}^{B}}{2}-Q_{2}^{A, B}
\end{aligned}
$$

where $Q_{1}^{A}$ (resp. $Q_{1}^{B}$ ) is actually the probability of sampling two genes (or alleles) identical in state (IIS) within population $A$ (resp. $B$ ) and $Q_{2}^{A, B}$ is the probability of sampling two IIS genes from $A$ and $B$. It directly follows from equations 1 and 2 that:

$$
F_{3}(A ; B, C)=\frac{1}{2}\left(Q_{1}^{A}+Q_{2}^{B, C}-Q_{2}^{A, B}-Q_{2}^{B, C}\right)
$$

and,

$$
F_{4}(A, B ; C, D)=\frac{1}{2}\left(Q_{2}^{A, C}+Q_{2}^{B, D}-Q_{2}^{A, C}-Q_{2}^{B, C}\right)
$$

The $Q_{1}$ and $Q_{2}$ probabilities, and hence the $F_{2}, F_{3}$ and $F_{4}$ parameters depend on both demographic parameters (i.e., population sizes, divergence times and other historical events) and marker polymorphism (i.e., their mutation rates and ascertainment process). For instance, under a simple pure-drift model with no mutation, if $p_{r}$ denotes the allele frequency of the ancestral population $R$ of two isolated populations $A$ and $B$ then $1-Q_{2}^{A, B}=\mathbb{E}\left[2 p_{A} p_{B} \mid p_{r}\right]=$ $2 p_{r}\left(1-p_{r}\right)$ which is the heterozygosity in $R$. Similarly, $1-Q_{1}^{A}=2 p_{r}\left(1-p_{r}\right) e^{-\tau_{A}}\left(\right.$ resp., $\left.1-Q_{1}^{B}=2 p_{r}\left(1-p_{r}\right) e^{-\tau_{B}}\right)$ where $\tau_{A}$ (resp. $\tau_{B}$ ) is the divergence time separating $R$ and $A$ (resp. B) on a diffusion timescale (i.e., in drift units of $\frac{1}{2 N_{e}}$ where $N_{e}$ is the effective population along the branch). As a consequence, the resulting estimates of $F_{2}, F_{3}$ and $F_{4}$ strongly depend on the underlying set of genetic markers and may not be compared across different datasets, even from the same populations. Various scaling procedure may actually helps in reducing this dependence. Scaling the $F_{2}$ with respect to the across population heterozygosity $1-Q_{2}$ leads to the standard definition of pairwise-population $F_{S T}$ in terms of IIS probabilities (Rousset, 2007; Hivert et al, 2018) which is also concordant with its original definition as the numerator of $F_{S T}$ (Reich et al, 2009; Peter, 2016):

$$
F_{S T}(A ; B) \equiv \frac{Q_{1}-Q_{2}^{A, B}}{1-Q_{2}^{A, B}}=\frac{F_{2}(A ; B)}{1-Q_{2}^{A, B}}
$$


where $Q_{1}=\frac{1}{2}\left(Q_{1}^{A}+Q_{1}^{B}\right)$ is the overall probability of sample two IIS genes within the same population (i.e., averaged over populations $A$ and $B$ ). Similarly, the scaled versions of the $F_{3}$ and $F_{4}$ statistics named $F_{3}^{\star}$ and $D$ respectively (Patterson et al, 2012; Green et al, 2010; Durand et al, 2011), can be expressed as: $F_{3}^{\star}(A ; B, C) \equiv$ $\frac{F_{3}(A ; B, C)}{1-Q_{1}^{A}}$ and $D(A, B ; C, D) \equiv \frac{F_{4}(A, B ; C, D)}{\left(1-Q_{2}^{A, B}\right)\left(1-Q_{2}^{C, D}\right)}$. To sum up, expressions of the $F$ and $D$ parameters as a function of $Q_{1}$ and $Q_{2}$ probability are finally defined as follows:

$$
\begin{gathered}
F_{2}(A ; B) \equiv \frac{Q_{1}^{A}+Q_{1}^{B}}{2}-Q_{2}^{A, B} \quad \text { and } F_{S T}(A ; B) \equiv \frac{F_{2}(A ; B)}{1-Q_{2}^{A, B}}=\frac{Q_{1}^{A}+Q_{1}^{B}-2 Q_{2}^{A, B}}{2\left(1-Q_{2}^{A, B}\right)} \\
F_{3}(A ; B, C) \equiv \frac{Q_{1}^{A}+Q_{2}^{B, C}-Q_{2}^{A, B}-Q_{2}^{A, C}}{2} \quad \text { and } F_{3}^{\star}(A ; B, C) \equiv \frac{F_{3}(A ; B, C)}{1-Q_{1}^{A}}=\frac{Q_{1}^{A}+Q_{2}^{B, C}-Q_{2}^{A, B}-Q_{2}^{A, C}}{2\left(1-Q_{1}^{A}\right)} \\
F_{4}(A, B ; C, D) \equiv \frac{Q_{2}^{A, C}+Q_{2}^{B, D}-Q_{2}^{A, D}-Q_{2}^{B, C}}{2} \quad \text { and } D(A, B ; C, D) \equiv \frac{F_{4}(A, B ; C, D)}{\left(1-Q_{2}^{A, B}\right)\left(1-Q_{2}^{C, D}\right)}=\frac{Q_{2}^{A, C}+Q_{2}^{B, D}-Q_{2}^{A, D}-Q_{2}^{B, C}}{2\left(1-Q_{2}^{A, B}\right)\left(1-Q_{2}^{C, D}\right)}
\end{gathered}
$$

\subsubsection{Unbiased parameter estimators from Pool-Seq read count and standard allele count data}

Let $y_{i j}$ be the allele count for an arbitrarily chosen reference allele and $n_{i j}$ the total number of sampled alleles (e.g., twice the number of genotyped individuals for a diploid species) at SNP $i$ in population $j$. For Pool-Seq read count data, the $y_{i j}$ 's are not observed and for a given pool $j$, it is assumed that $n_{i j}=n_{j}$ (the haploid sample size) for each and every SNP. We thus similarly defined $r_{i j}$ as the read counts for the reference allele and $c_{i j}$ the overall coverage observed at SNP $i$ in population $j$.

If allele count data are directly observed, unbiased estimators of the IIS probability within population $j\left(Q_{1, i}^{j}\right)$ and between a pair of populations $j$ and $k\left(Q_{2, i}^{j, k}\right)$ for a given SNP $i$ are:

$$
\widehat{Q}_{1, i}^{j}=\frac{y_{i j}\left(y_{i j}-1\right)+\left(n_{i j}-y_{i j}\right)\left(n_{i j}-y_{i j}-1\right)}{n_{i j}\left(n_{i j}-1\right)}=1-2 \frac{y_{i j}\left(n_{i j}-y_{i j}\right)}{n_{i j}\left(n_{i j}-1\right)}
$$

$$
\text { and } \quad \widehat{Q}_{2, i}^{j, k}=\frac{y_{i j} y_{i k}+\left(n_{i j}-y_{i j}\right)\left(n_{i k}-y_{i k}\right)}{n_{i j} n_{i k}}
$$

For Pool-Seq read count, unbiased estimators of $Q_{1, i}$ and $Q_{2, i}$ are similarly defined as (Hivert et al, 2018, eqns A37 and A40):

$$
\widehat{Q}_{1, i}^{j}=1-\frac{n_{j}}{n_{j}-1}\left(1-\frac{r_{i j}\left(r_{i j}-1\right)+\left(c_{i j}-r_{i j}\right)\left(c_{i j}-r_{i j}-1\right)}{c_{i j}\left(c_{i j}-1\right)}\right)=1-2 \frac{n_{j}}{n_{j}-1} \frac{r_{i j}\left(c_{i j}-r_{i j}\right)}{c_{i j}\left(c_{i j}-1\right)}
$$

and $\quad \widehat{Q}_{2, i}^{j, k}=\frac{r_{i j} r_{i k}+\left(c_{i j}-r_{i j}\right)\left(c_{i k}-r_{i k}\right)}{c_{i j} c_{i k}}$ 
Genome-wide estimates of all the parameters defined in eq. 3 above are then simply obtained from these unbiased estimators of IIS probabilities over all the I SNPs as:

$f_{2}(A ; B)=\widehat{F}_{2}(A ; B)=\frac{1}{2 I} \sum_{i=1}^{I}\left(\widehat{Q}_{1, i}^{A}+\widehat{Q}_{1, i}^{B}-2 \widehat{Q}_{2, i}^{A, B}\right)$

$f_{3}(A ; B, C)=\widehat{F}_{3}(A ; B, C)=\frac{1}{2 I} \sum_{i=1}^{I}\left(\widehat{Q}_{1, i}^{A}+\widehat{Q}_{2, i}^{B, C}-\widehat{Q}_{2, i}^{A, B}-\widehat{Q}_{2, i}^{A, C}\right)$

$f_{4}(A, B ; C, D)=\widehat{F}_{4}(A, B ; C, D)=\frac{1}{2 I} \sum_{i=1}^{I}\left(\widehat{Q}_{2, i}^{A, C}+\widehat{Q}_{2, i}^{B, D}-\widehat{Q}_{2, i}^{A, D}-\widehat{Q}_{2, i}^{B, C}\right) \quad$ and $\quad D(A, B ; C, D)=\frac{\sum_{i=1}^{I}\left(\widehat{Q}_{2, i}^{A, C}+\widehat{Q}_{2, i}^{B, D}-\widehat{Q}_{2, i}^{A, D}-\widehat{Q}_{2, i}^{B, C}\right)}{2 \sum_{i=1}^{I}\left(\left(1-\widehat{Q}_{2, i}^{A, B}\right)\left(1-\widehat{Q}_{2, i}^{C, D}\right)\right)}$

Similarly, the within-population heterozygosity $\widehat{h_{j}}$ for each population is simply estimated as:

$$
\widehat{h_{j}}=1-\frac{1}{I} \sum_{i=1}^{I} \widehat{Q}_{1, i}^{j}
$$

Importantly, for the three scaled parameters $F_{S T}, F_{3}^{\star}$ and $D$, multi-locus estimators consist of ratios of the numerator and denominator averages and not average of ratios (see e.g., Rousset, 2007; Patterson et al, 2012; Bhatia et al, 2013; Weir \& Goudet, 2017; Hivert et al, 2018). Hence, for pairwise $F_{S T}$, the above estimator is similar to the one described in Rousset (2007) for allele count data and identical to the alternative PID estimator described in Hivert et al (2018) for Pool-Seq read count data (so-called "Identity" method of the computeFST function from the poolfstat package).

\subsubsection{Block-Jackknife estimation of standard errors}

Following Reich et al (2009), standard-errors of genome-wide estimates of the different statistics are computed using block-jackknife (Kunsch, 1989; Busing et al, 1999) which consists of dividing the genome into contiguous chunks of a predefined number of SNPs and then removing each block in turn to quantify the variability of the estimator. For a given parameter $F$, if $n_{b}$ blocks are available and $\widehat{F_{b}}$ is the estimated statistics when removing all SNPs belonging to block $b$, the standard error $\widehat{\sigma_{F}}$ of the genome-wide estimator $\widehat{F}$ is computed as:

$$
\widehat{\sigma_{F}}=\sqrt{\frac{n_{b}-1}{n_{b}} \sum_{b=1}^{n_{b}}\left(\widehat{F_{b}}-\widehat{\mu_{F}}\right)^{2}}
$$


where $\widehat{\mu_{F}}=\frac{1}{n_{b}} \sum_{b=1}^{n_{b}} \widehat{F_{b}}$, which may be slightly different than the estimator obtained with all the $I$ markers since the latter may include SNPs that are not eligible for block-jackknife sampling (e.g., those at the chromosome or scaffolds boundaries). Finally, block-jackknife sampling may also be used to obtain estimates of the error covariance between two estimates $\widehat{F^{u}}$ and $\widehat{F^{v}}$ as:

$$
\widehat{\operatorname{Cov}}\left(\widehat{F^{u}} ; \widehat{F^{v}}\right)=\frac{n_{b}-1}{n_{b}} \sum_{b=1}^{n_{b}}\left(\widehat{F_{b}^{u}}-\widehat{\mu_{F}^{u}}\right)\left(\widehat{F_{b}^{v}}-\widehat{\mu_{F^{v}}}\right)
$$

For convenience, we here chose to specify the same number of SNPs for each block instead of a block size in genetic distance (Patterson et al, 2012; Reich et al, 2009). We therefore do not recourse to a weighted block-jackknife (Busing et al, 1999). In practice, this has little impact providing the distribution of markers is homogeneous along the genome and the amount of missing data is negligible.

\subsubsection{Admixture Graph fitting}

The approach implemented in the new version of poolfstat to fit admixture graphs from $f$-statistics is directly inspired from the one proposed by Patterson et al (2012) and implemented in the qpGraph software (see also Lipson, 2020). Briefly, let $\widehat{\mathbf{f}}$ the vector (of length $\frac{n_{l}\left(n_{l}-1\right)}{2}$ where $n_{l}$ is the number of graph leaves) of the estimated $f_{2}$ and $f_{3}$ statistics forming the basis of all the $f$-statistics (see above). Similarly, let $\mathbf{g}(\mathbf{e} ; \mathbf{a})=\mathbf{X}(\mathbf{a}) \times \mathbf{e}$ the vector of their expected values given the graph edge lengths vector $\mathbf{e}$ and an incidence matrix $\mathbf{X}(\mathbf{a})$, which summarize the structure of the graph given the vector a of proportions of all admixture events (for a tree-topology, $\mathbf{X}(\mathbf{a})$ only consists of 0 or 1). In poolfstat, $\mathbf{X}(\mathbf{a})$ is derived using simple operations from another $n_{l}$ by $n_{e}$ matrix (where $n_{e}$ is the number of graph edges) that specifies the weights of each edge along all the paths connecting the graph leaves to the root. It should be noticed that an admixture event is modeled as an instantaneous mixing of two populations $S_{1}$ and $S_{2}$ into a population $S$ directly ancestral to a child population $A$. An admixture event may thus be specified by i) one admixture rate $\alpha$ quantifying the relative $S_{1}$ and $S_{2}$ ancestry proportions ( $\alpha$ and $1-\alpha$ ) in population $S$; and ii) three edge lengths $e_{S \leftrightarrow A}$ for the branch connecting $S$ and $A$ and $e_{S_{1} \leftrightarrow \mathcal{G}}$ and $e_{S_{2} \leftrightarrow \mathcal{G}}$ for the branches connecting the two source populations to the rest of the graph $\mathcal{G}$. Yet, these three edge lengths are not identifiable and can only be estimated jointly in a single compound parameter (Pickrell \& Pritchard, 2012; Patterson et al, 2012; Lipson, 2020): $\zeta=\alpha^{2} \times e_{S_{1} \leftrightarrow \mathcal{G}}+(1-\alpha)^{2} \times e_{S_{2} \leftrightarrow \mathcal{G}}+e_{S \leftrightarrow A}$. Following Lipson (2020), this identifiability issue is solved by setting $e_{S_{1} \leftrightarrow \mathcal{G}}=e_{S_{2} \leftrightarrow \mathcal{G}}=0$ (i.e., nullifying the edges connecting the two source populations to the graph). Although it has no impact on the interpretation of the graph, this may overestimate the length of $e_{S \leftrightarrow A}$ (i.e, the divergence between 
the admixed population $A$ and its direct ancestor $S$ ). Proceeding this way differs from the choice made by Pickrell \& Pritchard (2012) in the Treemix package which consists, following our notations, of setting $e_{S \leftrightarrow A}=e_{S_{2} \leftrightarrow \mathcal{G}}=0$ if $\widehat{\alpha}>0.5$ and $e_{S \leftrightarrow A}=e_{S_{1} \leftrightarrow \mathcal{G}}=0$ otherwise.

We finally define $\mathbf{Q}$ as the $\frac{n_{l}\left(n_{l}-1\right)}{2}$ by $\frac{n_{l}\left(n_{l}-1\right)}{2}$ covariance matrix of the basis $f$-statistics estimated by blockjackknife. Graph fitting consists of finding the graph parameter values (ê and $\mathbf{a})$ that minimize a cost (score of the model) defined as:

$$
S(\mathbf{e} ; \mathbf{a})=(\widehat{\mathbf{f}}-\mathbf{g}(\mathbf{e} ; \mathbf{a}))^{\prime} \mathbf{Q}^{-1}(\widehat{\mathbf{f}}-\mathbf{g}(\mathbf{e} ; \mathbf{a}))=(\widehat{\mathbf{\Gamma}}-\mathbf{\Gamma X}(\mathbf{a}) \mathbf{e})^{\prime}(\widehat{\boldsymbol{\Gamma} \widehat{\mathbf{f}}}-\mathbf{\Gamma X}(\mathbf{a}) \mathbf{e})
$$

where $\boldsymbol{\Gamma}$ results from the Cholesky decomposition of $\mathbf{Q}^{-1}$ (i.e., $\mathbf{Q}^{-1}=\boldsymbol{\Gamma}^{\prime} \boldsymbol{\Gamma}$ ). Given admixture rates $\mathbf{a}, S(\mathbf{e} ; \mathbf{a})$ is quadratic in the edge lengths e (Patterson et al, 2012) leading us to rely on the Lawson-Hanson non-negative linear least squares algorithm implemented in the R package nnls (Lawson \& Hanson, 1995)) to estimate the vector $\hat{\mathbf{e}}$ that minimizes $S(\mathbf{e} ; \mathbf{a})$ (subject to the constraint of positive edge lengths). Full minimization of $S(\mathbf{e} ; \mathbf{a})$ is thus reduced to the identification of the admixture rates a which is performed using the L-BFGS-B algorithm implemented in the optim function of the R package stats (Nocedal \& Wright, 1999).

\subsubsection{Confidence Intervals and model fit assessment}

Assume $\widehat{\mathbf{f}} \sim \mathrm{N}(g(\hat{\mathbf{e}} ; \hat{\mathbf{a}}), \mathbf{Q})$, i.e., the vector of the basis $f$-statistics follows a multivariate normal distribution centered on the vector $g(\hat{\mathbf{e}} ; \hat{\mathbf{a}})$ specified by the fitted admixture graph parameters and the estimated error covariance matrix $Q$. The optimized score $S(\hat{\mathbf{e}} ; \hat{\mathbf{a}})$ then verifies $S(\hat{\mathbf{e}} ; \hat{\mathbf{a}})=-2 \log (L)-K$ where $L$ is the likelihood of the fitted graph and $K=n \log (2 \pi)+\log (|\mathbf{Q}|)$. This makes it straightforward to compute a BIC (Bayesian Information Criterion) for the fitted graph from the optimized score as:

$$
\mathrm{BIC}=S(\hat{\mathbf{e}} ; \hat{\mathbf{a}})+n_{\mathrm{par}} \log \left(\frac{1}{2} n_{l}\left(n_{l}-1\right)\right)-\frac{1}{2} n_{l}\left(n_{l}-1\right) \log (2 \pi)-\log (|\mathbf{Q}|)
$$

$B I C$ may then be useful to compare different fitted admixture graph topologies. When comparing two graphs $\mathcal{G}_{1}$ and $\mathcal{G}_{2}$ with $\mathrm{BIC}$ equal to $B I C_{1}$ and $B I C_{2}$ respectively, we have $\Delta_{12}=B I C_{2}-B I C_{1} \simeq 2 \log \left(B F_{12}\right)$ where $B F_{12}$ is the Bayes Factor associated to the comparison of the graphs $\mathcal{G}_{1}$ and $\mathcal{G}_{2}$ (Kass \& Raftery, 1995, eq. 9). We may further rely on the modified Jeffreys' rule proposed by Kass \& Raftery (1995) to assess to which extent the data support either the $\mathcal{G}_{1}$ or $\mathcal{G}_{2}$ graphs, with $\Delta_{12}>6$ (respectively $\Delta_{12}>10$ ) providing "strong" (respectively "very strong") evidence in favor of $\mathcal{G}_{1}$ (Supplementary Vignette V1). 
Moreover, the likelihood interpretation of the optimized score $S(\hat{\mathbf{e}} ; \hat{\mathbf{a}})=-2 \log (L)-K$ allows constructing confidence intervals (CI) for the fitted parameters of a given graph (i.e., elements of the $\mathbf{e}$ and a vectors) using the following uni-dimensional procedure. For a given parameter $v$ (either a edge length or an admixture rate), the difference $S_{v}(x)-S(\hat{\mathbf{e}} ; \hat{\mathbf{a}})$ (where $S_{v}(x)$ is the score when $v=x$ and all the other parameters are set to their best fitted values) can be interpreted as a likelihood-ratio test statistics following a $\chi^{2}$ distribution with one degree of freedom. Lower and upper boundaries $v_{\min }$ and $v_{\max }$ of the $95 \% \mathrm{CI}\left(\operatorname{such} S_{v}(x)-S(\hat{\mathbf{e}} ; \hat{\mathbf{a}})<3.84\right.$ for all $\left.v_{\min }<x<v_{\max }\right)$ may then simply be computed using a bisection method, as implemented in poolfstat.

Finally, a straightforward (but highly informative and recommended) approach to assess the fit of an admixture graph is to evaluate to which extent the $f$-statistics derived from the fitted admixture graph parameters $(g(\hat{\mathbf{e}} ; \hat{\mathbf{a}}))$ depart from the estimated ones (Patterson et al, 2012; Lipson, 2020). This can be summarized via a Z-score of residuals computed as $Z=\frac{f-\widehat{G}}{\sigma_{F}^{2}}$ where $\widehat{G}$ is a given fitted $f$-statistics; $f$ is its corresponding estimated values; and $\sigma_{F}^{2}$ the block-jackknife standard error. The presence of outlying Z-scores for at least one $f$-statistics (e.g., $|Z|>1.96$ at a $95 \%$ significance threshold) may suggest poor model fit while also providing insights into the leaves or graph edges that are the most problematic (Lipson, 2020).

\subsubsection{Scaling of branch lengths in drift units}

Admixture graph fitting results in estimated edge lengths on the same scale as $F_{2}$ which limits their interpretation, because they depend both on the overall level of SNP polymorphism and on their distance to the root (Patterson et al, 2012). Lipson et al (2013) proposed an empirical approach to rescale edge lengths on a diffusion timescale using estimates of overall marker heterozygosities within (i.e., $1-Q_{1}$ ) or across (i.e., $1-Q_{2}$ ) populations. The argument echoes the aforementioned interpretation of pairwise $F_{S T}$ as a scaled $F_{2}$. If $p_{C}$ and $p_{P}$ are the reference allele frequencies in a child node $C$ and its direct parent node $P$ and their divergence time (on a diffusion timescale) is $\tau_{C, P}=\frac{t}{N_{e}}$ (where $t$ is the branch length in generations), then conditional on $p_{C}, F_{2}(C ; P)=\left(1-e^{-\tau_{C, P}}\right) p_{C}\left(1-p_{C}\right)$ and $Q_{2}^{C, P}=1-2 p_{C}\left(1-p_{C}\right)$ leading to $F_{S T}^{C, P}=\frac{F_{2}(C ; P)}{1-Q_{2}^{C, P}}=\frac{1}{2}\left(1-e^{-\tau_{C, P}}\right)$ (i.e., $F_{S T}^{C, P} \simeq \frac{t}{2 N_{e}}$ when $\left.\tau_{C, P} \ll 1\right)$. Hence, the estimated graph edges length $\widehat{F_{2}}(C ; P)=\hat{e}_{\mathrm{P} \leftrightarrow \mathrm{C}}$ are scaled in units of drift by a factor equal to $=\frac{2}{\widehat{h_{P}}}$ where $\widehat{h_{P}}$ is the estimated heterozygosity (i.e., $1-\widehat{Q}_{1}^{P}$ ) in the (parent) node $P$. Rearranging equation 2 and using $Q_{2}^{C, P}=Q_{1}^{P}$ (conditional on $p_{P}$ ) shows that $h_{P}=F_{2}(C ; P)+h_{C}$, where $h_{C}=\left(1-Q_{1}^{C}\right)$ is the heterozygosity of the child node $C$. Hence, all the node heterozygosities can be inferred iteratively from the leaves to the root along the admixture graph using the leave heterozygosities (directly estimated from the data) and the fitted edge lengths (Lipson et al, 2013) 


\subsubsection{Admixture Graph construction}

Comprehensive exploration of the space of possible admixture graphs rapidly becomes impossible even for a moderate number of populations. We implemented in poolfstat different heuristics to facilitate admixture graph construction based on a supervised approach (see Supplementary Vignette V1 for details). First the add.leaf function allows exploring all the possible connections of a new population to an existing admixture graph. If $n_{e}$ is the number of edges of the admixture graphs, $n_{e}+1$ possible graphs connecting the new leaf with a non-admixed edges (i.e., including a new rooting with the candidate leaf as an outgroup) and $\frac{1}{2} n_{e}\left(n_{e}-1\right)-1$ connecting the new leaf with a two-way admixture event are then tested. Note that an admixture between the two root edges is excluded from the exploration since it results in a singular model. More generally, the different possible graphs are always checked for singularity by empirically verifying that the rank of the model incidence matrix $\mathbf{X}(\mathbf{a})$ is equal to the number of edges to fit. The different fitted graph can then be ranked according to their BIC, the graph with the lowest BIC having the strongest support.

The graph.builder function allows a larger exploration of the graph space by successively adding several leaves in a given order to an existing admixture graph. At each step of the process, a heap stores the best resulting graph together with some intermediary sub-optimal graphs based on their BIC. After initializing the heap with some graph (or a list of graphs), the add.leaf function is called to evaluate, for each candidate leaf in turn, all its possible connections (with non-admixed or admixed edges) to all the graphs stored in the heap. Among the obtained graphs, the one with the lowest $B I C$ together with those with a $B I C$ within a given $\Delta_{B I C}\left(\Delta_{B I C}=6\right.$ by default) are included in a newly generated heap. If the resulting heap contains more than a predefined number of graphs $n_{g}^{\max }\left(n_{g}^{\max }=25\right.$ by default), only the $n_{g}^{\max }$ graphs with the lowest $B I C$ are finally kept in the heap of graphs to be used for the addition of the next leaf. Although helpful, such heuristic should be used cautiously and we recommend to only try adding a small number of populations (i.e., $\leq 5$ ) to an existing graph. One also needs to evaluate different orders of population inclusion (Supplementary Vignettes V1 and V2).

It is also critical to start these supervised procedures with graphs that are representative of the whole history of the populations under study and not too unbalanced with respect to the candidate leaves. In particular, starting with a small tree of closely related populations which are distantly related to the candidate leaves must be avoided. When prior knowledge about the history of the investigated population is limited (which is usually the case), Lipson $e t$ al (2013) proposed to start admixture graph construction with a scaffold tree of populations displaying no evidence of admixture. As in the absence of admixture, $F_{2}$ statistics are expected to be additive along the paths of the (binary) population tree, its unrooted topology and branch lengths may simply be inferred with a neighbor-joining 
algorithm. In poolfstat, we implemented two functions that allow i) identifying candidate sets of unadmixed populations among all the genotyped ones (find.tree.popset); and ii) building rooted neighbor-joining tree (rooted.njtree.builder). Briefly, find.tree.popset implements a procedure consisting of i) discarding all the populations showing at least one significant three-population test (i.e., displaying a negative $F_{3} \mathrm{Z}$-score lower than -1.65 by default) among all the possible ones; and ii) identifying via a greedy algorithm the largest sets of populations for which all the possible quadruplets pass the four-population test of treeness (i.e., with an absolute $F_{4} \mathrm{Z}$-score lower than 1.96 by default). The rooted.njtree.builder function builds a scaffold tree from a candidate set of (presumably) unadmixed populations using the $\mathrm{nj}$ function from the ape package (Paradis $e t a l$, 2004) and then compare the consistency of population heterozygosities between the partitions of the tree to root it (Lipson et al, 2013). Note that this latter procedure may be sensitive to long-branch attraction and should thus be used carefully when including highly divergent populations.

\subsection{Overview of the new poolfstat package}

Tables 1 and 2 describe the main objects and functions implemented in our new version (v2.0.0) of the R package poolfstat publicly available from the CRAN repository (https://cran.r-project.org/web/packages/ poolfstat/index.html). In-depth analyses of two Pool-Seq and allele count simulated datasets (see below) are described for illustration purposes in the package vignette provided as Supplementary Vignette V1. Detailed documentation page of the different objects and functions can also be directly accessed from an R terminal with poolfstat loaded using the help function (or the ? operator).

The package includes several functions to parse allele count (e.g., genotreemix2countdata) or Pool-Seq (e.g., vcf2pooldata) input data stored in various formats commonly used in population genomics studies (Table 2). These functions allow to clearly distinguish these two different types of data by producing objects of either the so-called countdata (for allele count) or pooldata (for Pool-Seq data) classes (Table 1). This step is critical to further rely on the appropriate unbiased estimators for the $F$ and $D$ parameters. Some functions allow to perform subsequent manipulation of the input data, for instance to only consider some of the populations or to remove SNPs according to various criteria (Table 2).

The three functions computeFST, compute.pairwiseFST and compute.fstats implement the unbiased estimators of the different $f-, D$ - and within-population heterozygosities (based on allele IIS probabilities within and between pairs of populations) together with block-jackknife estimation of their standard errors. Importantly, these three functions automatically detect the appropriate estimators given the type of data (either allele or Pool- 


\begin{tabular}{|l|l|}
\hline S4 object & Description \\
\hline countdata & Standard allele count data (i.e., obtained from individual genotyping or sequencing data) \\
\hline pooldata & Pool-Seq read count data \\
\hline pairwisefst & $\begin{array}{l}\text { Store pairwise } F_{S T} \text { estimates. This object is generated by the compute.pairwiseFST func- } \\
\text { tion. Estimates can be conveniently visualized with the heatmap or plot functions, the } \\
\text { latter interfacing the plot_fstats function of poolfstat. }\end{array}$ \\
\hline fstats & $\begin{array}{l}\text { Store } F_{2}, \text { pairwise } F_{S T}, F_{3}, F_{3}^{\star}, F_{4} \text { and } D \text { estimation results. This object is generated by the } \\
\text { compute.fstats function. Estimates can be conveniently visualized with the heatmap or } \\
\text { plot functions, the latter interfacing the plot_fstats function of poolfstat. }\end{array}$ \\
\hline graph.params & $\begin{array}{l}\text { Represent a population tree or an admixture graph and its parameter. This object is generated } \\
\text { by the generate.graph.params function. The graph can be visualized with the plot } \\
\text { function that interfaces the grViz function from the DiagrammeR package (Iannone, 2020). }\end{array}$ \\
\hline & $\begin{array}{l}\text { Represent a population tree or an admixture graph and its underlying fitted parameters as } \\
\text { obtained from the fit.graph or other fitting functions. The graph can be visualized with the } \\
\text { plot function that interfaces the grViz function from the DiagrammeR package (Iannone, } \\
\text { 2020). }\end{array}$ \\
\hline
\end{tabular}

Table 1. Description of the main S4 objects of the poolfstat package. ${ }^{\dagger}$ Object already existing in the first poolfstat version.

Seq read counts) according to the input object class (either countdata or pooldata). For the estimation of $F_{S T}$, the computeFST and compute.pairwiseFST also implement (by default) estimators based on an Analysis of Variance framework that correspond to those developed by Weir (1996) for allele count data and by Hivert et al (2018) for Pool-Seq data.

The fit.graph function implements the approach described above to estimate the parameters (i.e., edge lengths and admixture rates) of an admixture graph that is stored in a graph.params object (Table 1). Such objects can be generated with the generate. graph. params function (Table 2) to include the target basis $f$-statistics and the error covariance matrix (denoted above $\widehat{\mathbf{f}}$ and $\mathbf{Q}$, respectively) estimated with compute. fstats (stored in an fstats object) and to specify the topology and the parameters of the admixture graph. Note that the graph.params2symbolic.fstats function allows exploring in details the properties of an admixture graph specified by a graph. params object by deriving a symbolic representation of all the $F_{2}, F_{3}, F_{4}$ and the model equations (see above) by internally relying on the Ryacas package for symbolic computation (Andersen \& Højsgaard, 2019). The fit. graph function then produced an object of class fitted.graph that includes the estimated edge lengths (in $F_{2}$ and also optionally in drift units) and admixture proportions together with (optionally) their 95\% CI. For model fit assessment purposes, fitted. graph objects also include the BIC and Z-score of the residuals of the fitted basis $f$-statistics. Such a comparison can (and should) be generalized to all the $f_{2}, f_{3}$ and $f_{4}$ statistics (not just the ones forming the basis) using compare . fitted. fstats jointly applied to a fitted.graph and a fstats ob- 
bioRxiv preprint doi: https://doi.org/10.1101/2021.05.28.445945; this version posted May 30, 2021. The copyright holder for this preprint (which

was not certified by peer review) is the author/funder, who has granted bioRxiv a license to display the preprint in perpetuity. It is made available under aCC-BY-NC-ND 4.0 International license.

\begin{tabular}{|c|c|c|c|c|c|c|c|c|c|c|c|c|c|c|c|c|c|c|c|c|c|}
\hline & 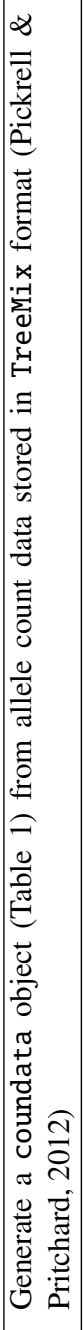 & 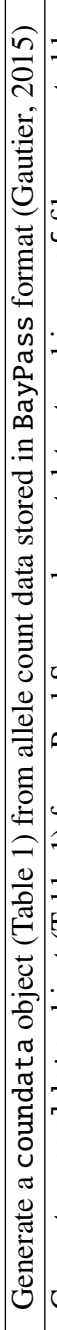 & 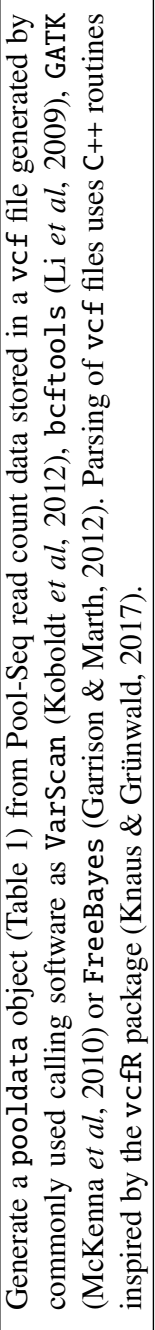 & 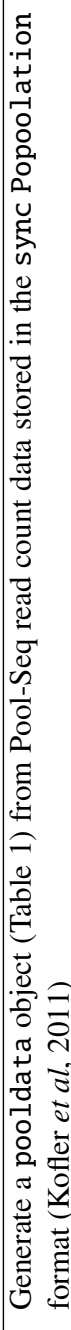 & 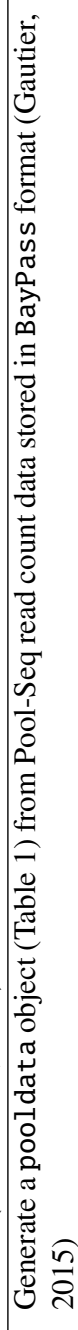 & 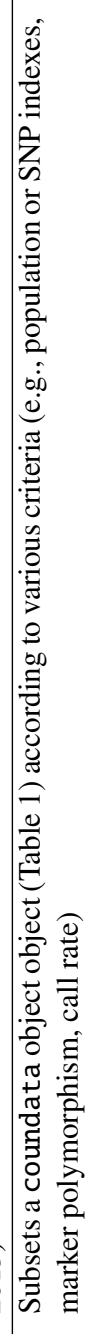 & 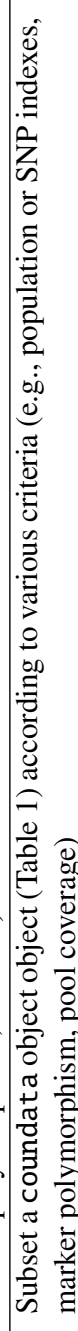 & 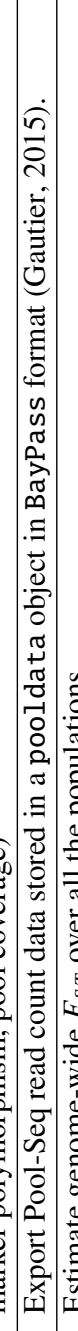 & 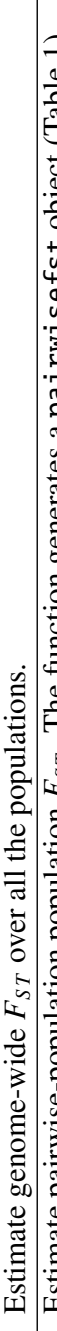 & 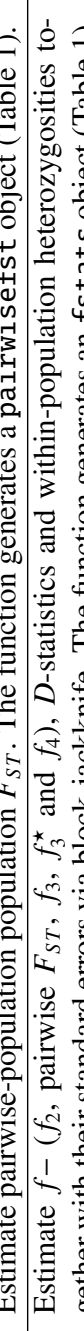 & 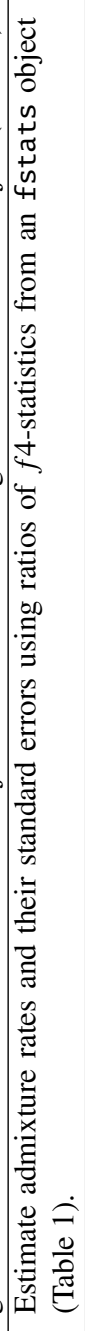 & 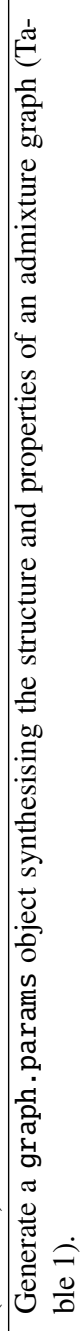 & 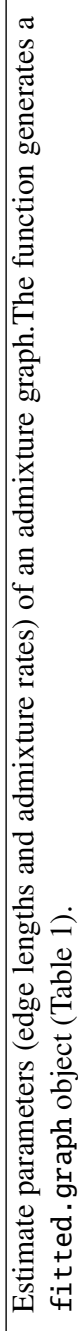 & 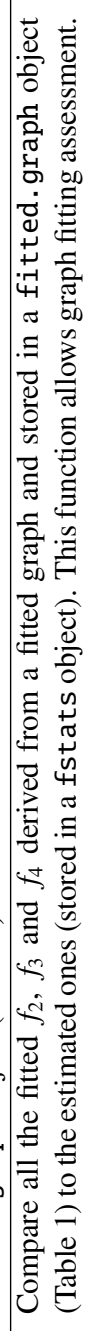 & 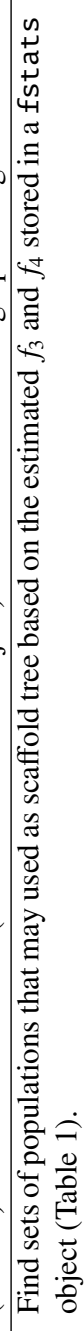 & 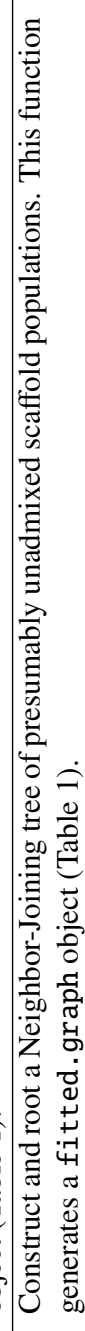 & 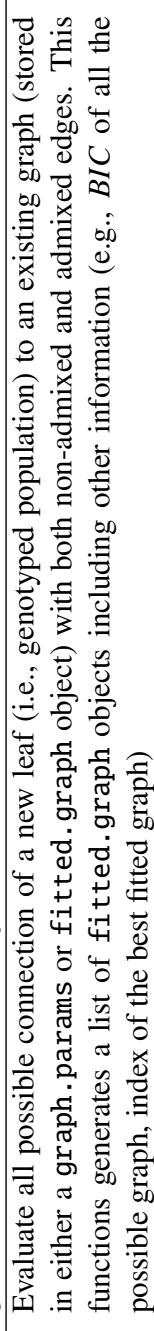 & 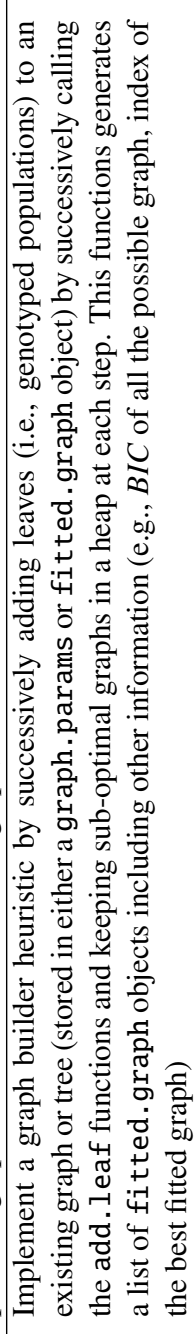 & & 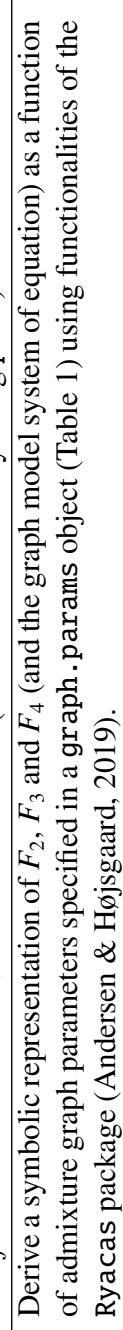 & 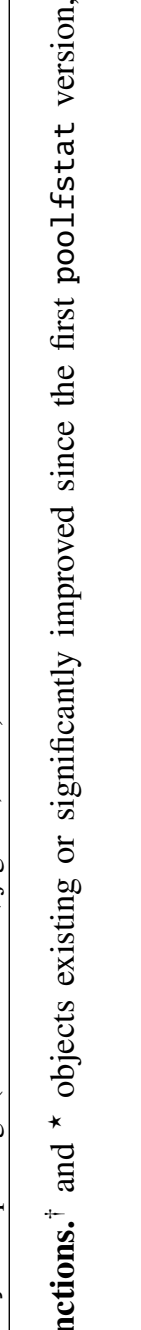 \\
\hline 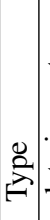 & 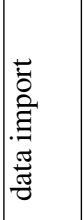 & 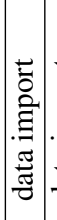 & 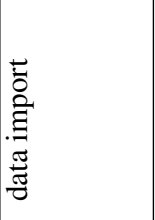 & . & 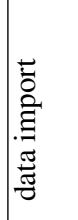 & 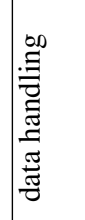 & 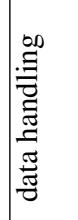 & 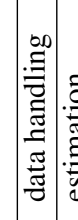 & 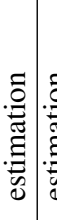 & 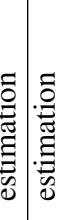 & 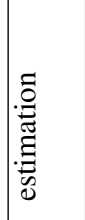 & 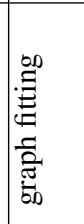 & 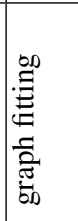 & 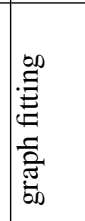 & 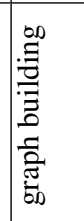 & 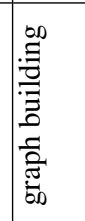 & 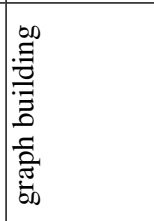 & 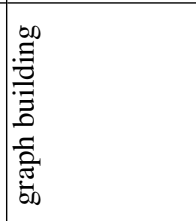 & & כ. & 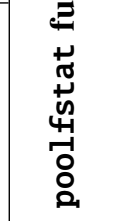 \\
\hline & 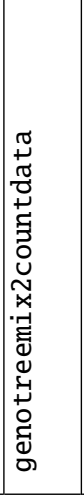 & 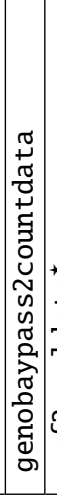 & 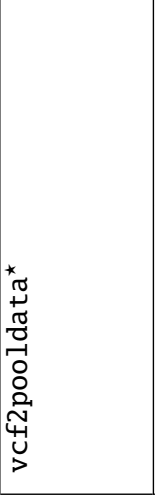 & 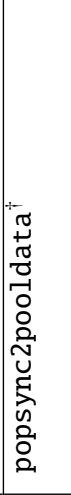 & 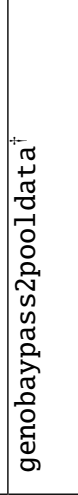 & 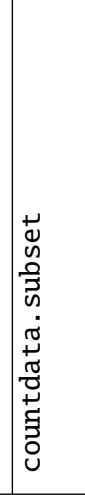 & 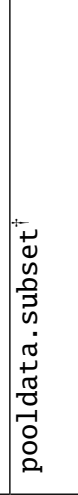 & 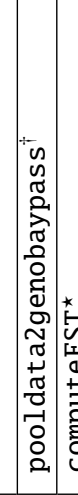 & 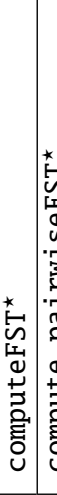 & 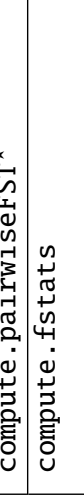 & 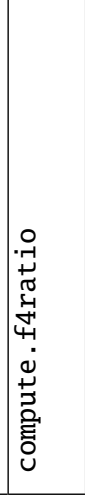 & 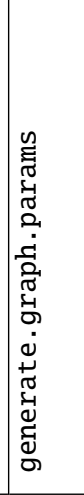 & 岁 & 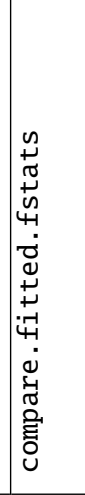 & 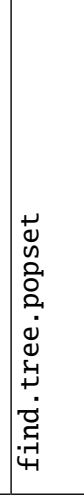 & 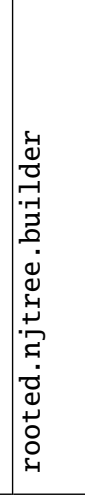 & & ق & & 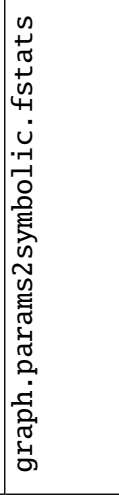 & 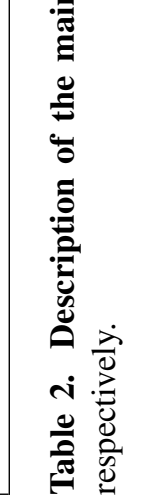 \\
\hline
\end{tabular}


jects. Notice that we developed for comparison purposes a function named graph.params2qpGraphFiles to export admixture graph specification and their underlying estimated basis $f$-statistics (both stored in a graph.params object) into qpGraph format (Patterson et al, 2012), allowing independent fitting based on the same estimated statistics to be carried out with this later program.

The poolfstat package includes several functions to assist construction of admixture graphs. As mentioned in the previous section, the find.tree.popset and rooted.njtree.builder functions allow to identify and build rooted tree(s) of scaffold of (presumably) unadmixed populations that may be used as starting graph(s). Besides, the add. leaf and graph. builder functions implement the above described heuristic to extent an existing graph (or tree) by adding one or several leaves (i.e., genotyped populations). These functions generate a list of fitted.graph objects together with other information that may be helpful for graph comparison (e.g., BIC of all the graphs or index of the best fitted graph).

Finally, as detailed and exemplified in the Supplementary Vignette V1, fitted graphs (stored in fitted.graph objects) and non-fitted graphs (stored in graph. params objects) can be directly and conveniently plotted with the plot function which internally interfaces the grViz function from the DiagrammeR package (Iannone, 2020).

\subsection{Data analyses}

\subsubsection{Simulation study}

Genetic data for a total of 150 diploid individuals belonging to six different populations ( $\mathrm{n}=25$ individuals per populations) related by the demographic scenario depicted in Figure 1 were simulated using the msprime coalescent simulator (Kelleher et al, 2016) with the following command:

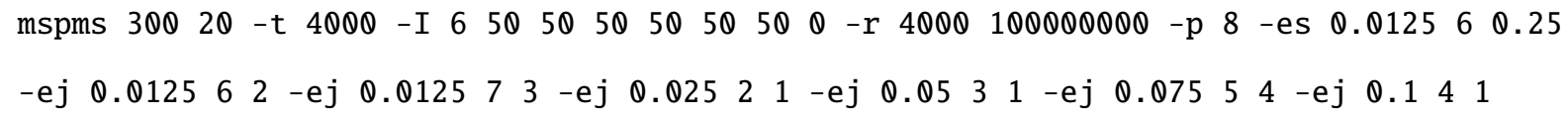

Each genome thus consisted of 20 independent chromosomes of $L=100 \mathrm{Mb}$ assuming a scaled chromosomewide recombination rate of $\rho=4 L N_{e} r=4,000$ as expected for instance in a population of constant diploid effective size of $N_{e}=10^{3}$ when the per-base and per-generation recombination rate is $r=10^{-8}$ (i.e., one cM per $\mathrm{Mb}$ ). The scaled chromosome-wide mutation rate was set to $\theta=4 L N_{e} \mu=4,000$ which is also the expected nucleotide diversity in a population with $N_{e}=10^{3}$ at mutation-drift equilibrium when the per-base mutation rate is $\mu=10^{-8}$. A total of 250 independent genotyping datasets were simulated and each was subsequently processed to generate 32 different types of datasets corresponding to: 


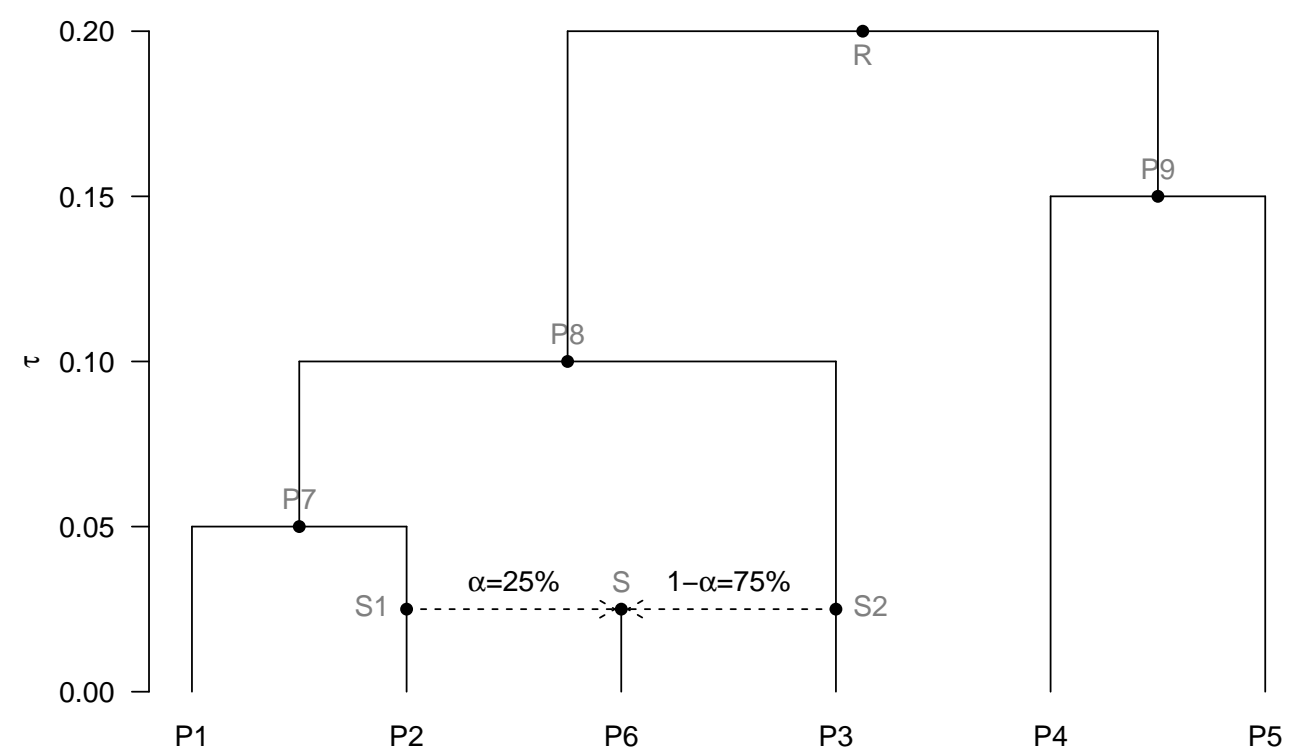

Figure 1. Simulated scenario relating six sampled populations. The population $P 6$ derived from a population $S$ which is admixed between two ancestral sources $(S 1$ and $S 2)$ directly related to populations $P 2$ and $P 3$ and contributing to $\alpha=25 \%$ and $1-\alpha=75 \%$ of its genome, respectively. The branch lengths are in a diffusion timescale i.e., with $\tau=\frac{t}{2 N_{e}}$ under a pure-drift model of divergence (where $t$ is the number of non-overlapping generations and $N_{e}$ the average diploid effective population sizes along the branch). The names of the internal node populations (not sampled) are represented in grey.

- Two standard allele count datasets (namely $\mathrm{AC}_{m \geq 1 \%}$ and $\mathrm{AC}_{m \geq 5 \%}$ ) obtained by simple counting of the simulated individual (haploid) genotypes for each population (i.e., assuming Hardy-Weinberg equilibrium within population) and removing SNPs with a Minor Allele Frequency (MAF) computed over all the individuals lower than $1 \%$ (for $\mathrm{AC}_{m>1 \%}$ datasets) or $5 \%$ (for $\mathrm{AC}_{m>5 \%}$ datasets)

- Thirty Pool-Seq datasets (coded as PS $\lambda_{m>m_{t}}^{\epsilon=\epsilon}$ ) for i) five different average sequencing coverages $\lambda$ (equal to $30,50,75,100$ or 200 reads; a 30X Pool-Seq coverage representing a lower limit for population genomics studies); ii) two different MAF thresholds $m_{t}$ of $1 \%$ and $5 \%$ (MAF being estimated on the read counts over all the pools); and iii) three different sequencing error rates $\epsilon$ of 0 (no error), $1 \%$ and $2.5 \%$ the two latter being representative of Illumina sequencers (Glenn, 2011).

Pool-Seq datasets were simulated from the $\mathrm{AC}_{m \geq 1 \%}$ allele count datasets following a procedure similar to that described in Hivert et al (2018). Briefly, the vector $\boldsymbol{r}_{i j}=\left\{r_{i j k}\right\}$ of read counts at SNP position $i$ in population $j$ for the nucleotide $k$ (where by convention $k=1$ and $k=2$ for the derived and ancestral alleles respectively) was sampled from a Multinomial distribution parameterized as: 


$$
\boldsymbol{r}_{i j} \sim \operatorname{Multin}\left(\left\{\frac{y_{i j}}{n_{j}}\left(1-\frac{\epsilon}{3}\right)+\left(1-\frac{y_{i j}}{n_{j}}\right) \frac{\epsilon}{3} ;\left(1-\frac{y_{i j}}{n_{j}}\right)\left(1-\frac{\epsilon}{3}\right)+\frac{y_{i j}}{n_{j}} \frac{\epsilon}{3} ; \frac{\epsilon}{3} ; \frac{\epsilon}{3}\right\} ; c_{i j}\right)
$$

where $y_{i j}$ is the derived allele count for $\mathrm{SNP} i$ in population $j$ (from the corresponding $\mathrm{AC}_{m>1 \%}$ dataset); $n_{j}$ is the haploid sample size of population $j$ (here $n_{j}=50$ for all $j$ ); and $c_{i j}=\sum_{k=1}^{k=4} r_{i j k}$ is the overall read coverage. To introduce variation in read coverages across pools and SNPs, each $c_{i j}$ was sampled from a Poisson distribution with a parameter $\lambda$ (the target Pool-Seq mean coverage). When $\epsilon=0$, only reads for the derived $(k=1)$ or ancestral $(k=2)$ alleles can be generated and the above Multinomial sampling actually reduces to a Binomial sampling following $r_{i j 1} \sim \operatorname{Bin}\left(\frac{y_{i j}}{n_{j}} ; c_{i j}\right)$ (and $r_{i j 2}=c_{i j}-r_{i j 1}$ ). However, when $\epsilon>0$, sequencing errors might lead to non-null read counts for the two other alleles leading to tri- or tetra- allelic SNPs. Morevover, sequencing errors may also introduce spurious additional variation by generating false SNPs at monomorphic sites. To account for the latter, read count vectors $\boldsymbol{r}_{i^{\prime} j}$ for all the $2 \times 10^{9}-I$ monomorphic positions $i^{\prime}$ (where $I$ is the number of SNPs observed in the considered $\mathrm{AC}_{m>1 \%}$ dataset) were sampled as $\boldsymbol{r}_{i^{\prime} j} \sim \operatorname{Multin}\left(\left\{1-\frac{\epsilon}{3} ; \frac{\epsilon}{3} ; \frac{\epsilon}{3} ; \frac{\epsilon}{3}\right\} ; c_{i^{\prime} j}\right)$ with coverages $c_{i^{\prime} j}$ sampled from a Poisson distribution (as $c_{i j}$ for polymorphic positions). Yet, as usually done with empirical datasets, we applied a minimum read count filtering step consisting of disregarding all the alleles with less than 2 observed reads (over all the populations). Only bi-allelic SNPs passing the overall MAF threshold $m_{f}$ were finally retained in the final PS $\lambda_{m \geq m_{t} \%}^{\epsilon=\epsilon}$ datasets.

Analyses of the simulated data were carried out with poolfstat (Supplementary Vignette V1). Briefly, each msms simulated dataset was converted into an $\mathrm{AC}_{m \geq 1 \%}$ dataset in TreeMix format (Pickrell \& Pritchard, 2012) further imported into a countdata object with genotreemix2countdata (Tables 1 and 2) and used to generate each corresponding $\mathrm{AC}_{m \geq 5 \%}$ dataset using countdata. subset. To improve computational efficiency, the different $\mathrm{PS} \lambda_{m>m_{t} \%}^{\epsilon=\epsilon}$ Pool-Seq datasets were generated from the $\mathrm{AC}_{m \geq 1 \%}$ countdata objects in the form of pooldata object using custom functions (not included in the package) coded in $\mathrm{C}++$ and integrated within R using Rcpp (Eddelbuettel, 2013). In addition, to evaluate the impact of the (bad) practice consisting of analyzing Pool-Seq data as if they were allele count data (i.e., overlooking the sampling of reads from individual genes of the pool), we also created "fake" countdata objects from the different pooldata objects. We then used default options (unless otherwise stated) of i) computeFST to estimate genome-wide $F_{S T}$ over all the populations; ii) compute. fstats to estimate all the $f$ - and $D$ - statistics; iii) compute. f4ratio to estimate admixture proportions; and iv) fit.graph to estimate the admixture graph parameters (Table 2). As the number of SNPs was variable across the different simulated datasets, we adjusted the number of successive SNPs defining a block for block-jackknife estimation of standard 
errors by dividing the total number of available SNPs by 500. This thus resulted on average in 490 blocks of 4.1 $\mathrm{Mb}$ over the genome for all the analyzed datasets (the simulated genomes consisting of 20 chromosomes). Note that the parameter estimates were always taken as the block-jackknife mean values rather than estimates over all SNPs (i.e., including those in the chromosome ends). In practice, the differences between the two are insignificant (e.g., Supplementary Vignette V1).

For validation purposes, we also analyzed the $250 \mathrm{AC}_{m \geq 1 \%}$ datasets with programs from the AdmixTools suite (Patterson et al, 2012) after conversion to the appropriate input format using custom awk scripts. More specifically, we ran qpfstats (v. 200) to estimate the 15 basis $f$-statistics, i.e., taking $P 1$ as the reference population, the five $f_{2}$ of the form (P1,Px) and the ten $f_{3}$ of the form (P1;Px,Py) (where $x=2, \ldots, 6$ and $y=3, \ldots, 6$ with $y \neq x$ ) and their corresponding error covariance matrix. Default options were considered except for the disabling of the scaling of estimated values (using option -1 1) to facilitate their comparison with poolfstat estimates. We also ran with default options qp3Pop (v. 650) to estimate $f_{3}^{\star}$ for all the 60 possible triplet configurations and qpDstat (v. 970 ) to estimate the $D$-statistics for all the 45 possible quadruplet configurations together with their associated $Z$-scores. By default, these three programs define blocks of $5 \mathrm{cM}$ to implement the (weighted) block-jackknife procedure. As we here converted the simulated SNP positions from $\mathrm{Mb}$ to $\mathrm{cM}$ assuming one $\mathrm{cM}$ per $\mathrm{Mb}$ (see above), the sizes of the 400 blocks was thus about $20 \%$ than for poolfstat analyses.

\subsubsection{Analysis of a real Drosophila suzukii Pool-Seq data}

The spotted wing drosophila, Drosophila suzukii, represents an attractive model to study biological invasion and hence recent historical and demographic history. Native to South East Asia, this pest species was first observed outside its native range in Hawaii in 1980, and later rapidly invaded America and Europe simultaneously between 2008 and 2013 (Fraimout et al, 2017). Using DNA sequences and microsatellite markers, Adrion et al (2014) and Fraimout et al (2017) deciphered the routes taken by D. suzukii during its worldwide invasion. Both studies showed that America and European populations globally represent separate invasion routes with different native source populations. Olazcuaga et al (2020) recently generated Pool-Seq genomic data from 22 worldwide population samples to detect genetic variants associated with the historical status (i.e. invasive versus native) of the sampled populations. We here focused our illustration on 14 Pool-Seq data from this study (with 50 to 100 diploïd individuals per pool) for populations representative of the Asian native area (six populations), Hawaii (one population) and the invaded continental America (seven populations), where the species was first observed in 2008 on the Western coast of the USA (around Watsonville, CA; Figure 2A). Beside native populations, we have restricted 
our analysis to the American continent because the invasion of this area is characterized by multiple admixture events between different source populations (Fraimout et al, 2017), which makes it an appealing situation to evaluate the power and the limitation of poolfstat analyzes. Moreover, 13 of our 14 population samples consist of individuals originating from the same sites (albeit sometimes collected at different dates for some pools; Table 2 in Supplementary vignette V2) as those genotyped at 25 microsatellite markers and analyzed with an Approximate Bayesian Computation Random Forest (ABC-RF) approach to infer the routes of invasion on a worldwide scale by Fraimout et al (2017).

To allow for complete reproduction (and exploration) of our analyses, all the command lines used to analyze the D. suzukii Pool-Seq dataset are described in the Supplementary vignette V2. Briefly, we combined the 14 (bam) files, obtained by Olazcuaga et al (2020) after aligning the 14 Pool-Seq data onto the latest near-chromosome scale D. suzukii assembly (Paris et al, 2020), into an mpileup file using SAMtools 1.9 with options -q 20 -Q20 (Li et al, 2009). Variant calling was then performed using VarScan mpileup2snp v2.3.4 Koboldt et al (2012) run with options --min-coverage 10 --min-avg-qual 25 --min-var-freq 0.005 --p-value 0.5 (i.e., with very loose criteria). After discarding positions mapping to non-autosomal contigs (Paris et al, 2020), the resulting vcf file was parsed with the vcf2pooldata function of poolfstat with default options except for i) the overall MAF threshold (computed from read counts) that was set to 5\%; and ii) the minimal read coverage for each pool that was set to 50. The resulting pooldata object was further filtered with pooldata.subset to discard i) all positions with a coverage higher than the 99th coverage percentile within at least one pool; and ii) discard all SNPs with MAF $<5 \%$ over all the populations from the native area to favor ancestral SNPs. The final dataset then consisted of read counts for 1,588,569 bi-allelic SNPs with a median read coverage varying from 64 (US-Sok) to 95 (CN-Bei and US-Haw) among the 14 pools (Table 2 in the Supplementary Vignette V2). We defined blocks of 10,000 consecutive SNPs for block-jackknife estimation of standard errors leadint to a total of 145 blocks of 698 $\mathrm{kb}$ on average (varying from $414 \mathrm{~kb}$ to $2.03 \mathrm{Mb}$ ). Hence, most analyses actually relied on 1,450,000 SNPs that mapped to the 15 largest contigs of the assembly (totaling $116 \mathrm{Mb}$ ). In other words, SNPs mapping to the smallest (and less reliable) contigs were discarded in addition to the few ones mapping to the end of the 15 retained contigs. 
bioRxiv preprint doi: https://doi.org/10.1101/2021.05.28.445945; this version posted May 30, 2021. The copyright holder for this preprint (which was not certified by peer review) is the author/funder, who has granted bioRxiv a license to display the preprint in perpetuity. It is made available under aCC-BY-NC-ND 4.0 International license.

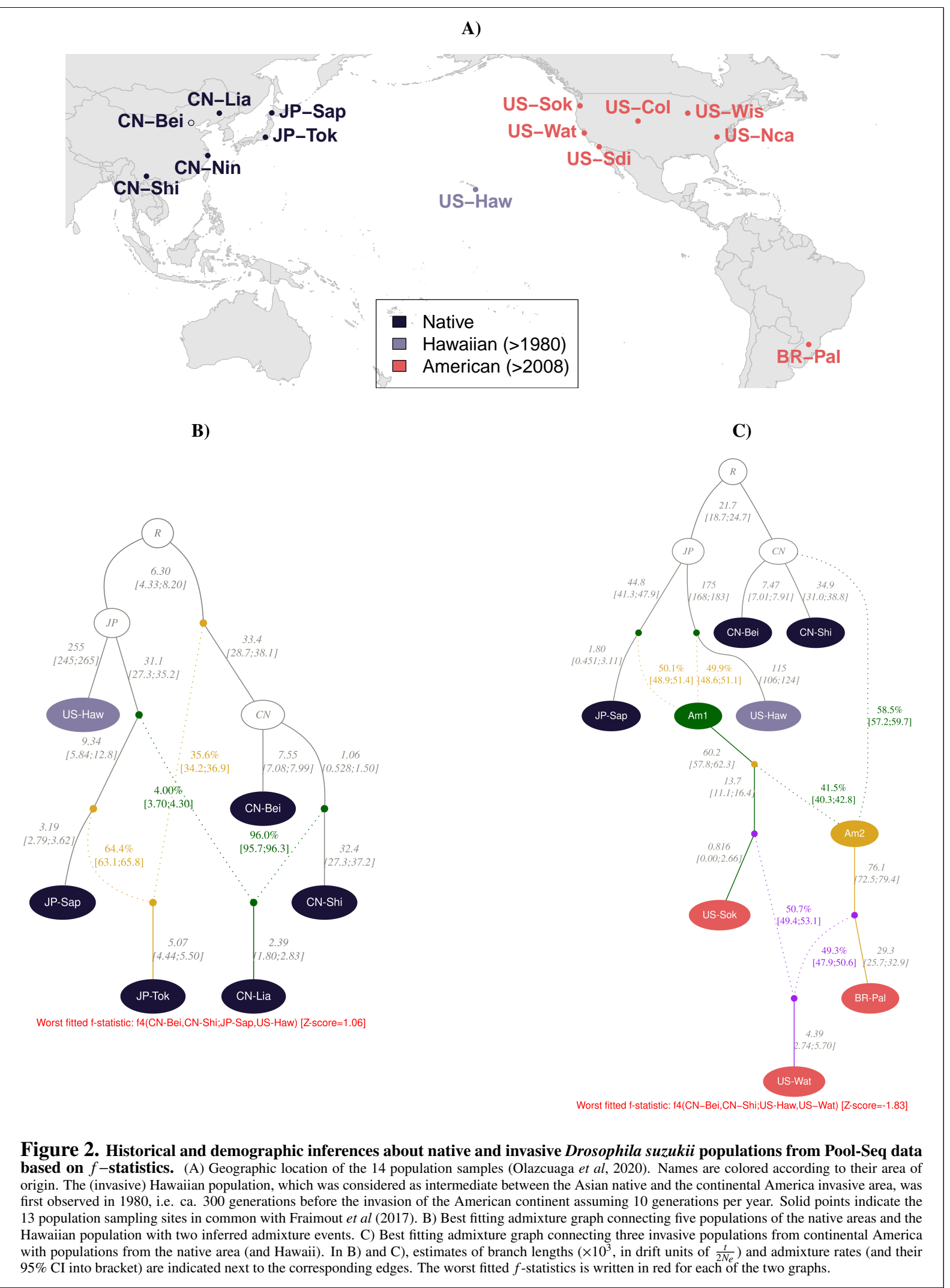




\section{Results}

\subsection{Evaluation of poolfstat on simulated data}

Historical and demographic inference based on $f$ - and $D$ - statistics has already been extensively evaluated in previous studies (e.g., Patterson et al, 2012; Lipson et al, 2013; Peter, 2016). Therefore, the purpose of our simulation study was essentially threefold: i) to validate the estimators implemented in poolfstat by comparing, for allele count data, with those obtained with the reference AdmixTools suite (Patterson et al, 2012); ii) to evaluate the performance of the estimators for Pool-Seq data as a function of read coverage and sequencing errors; and iii) to provide example datasets with known ground truth for illustration purposes.

\subsubsection{Description of the simulated datasets}

We simulated 250 genetic datasets for six populations (named $P 1$ to $P 6$ ) each consisting of 25 diploid individuals and that were historically related by the admixture graph represented in Figure 1 (Material and Methods). Each of these datasets was further used as template to generate 2 allele count datasets (applying 1\% or 5\% threshold on the overall MAF for $\mathrm{AC}_{m>1 \%}$ and $\mathrm{AC}_{m>5 \%}$ datasets respectively) and to simulate 30 Pool-Seq datasets with five different mean read coverages $(\lambda \in\{30 ; 50 ; 75 ; 100 ; 200\})$; three sequencing error rates $\left(\epsilon \in\left\{0 ; 10^{-3} ; 2.5 \times 10^{-3}\right\}\right)$ and two MAF (computed over all read counts) thresholds (referred to as PS $\lambda_{m>1 \%}^{\epsilon=\epsilon}$ and $\operatorname{PS} \lambda_{m>5 \%}^{\epsilon=\epsilon}$ for $1 \%$ and 5\% MAF thresholds, respectively). This thus lead to a total of 8,000 simulated datasets. The average number of available SNPs and false SNPs (for PS $\lambda_{m \geq m_{t} \%}^{\epsilon=1 \%}$ and $\operatorname{PS} \lambda_{m>m_{t} \%}^{\epsilon=2.5 \%}$ datasets) is given in Table S1 for each of the 32 different types of datasets and represented as a function of the mean coverages $\lambda$ and MAF thresholds in Figure S1.

Overall, 471,919 SNPs and 240,369 SNPs were available on average for allele count datasets at the $1 \%$ $\left(\mathrm{AC}_{m>1 \%}\right)$ and 5\% ( $\left.\mathrm{AC}_{m>5 \%}\right)$ MAF thresholds respectively consistent with the L-shaped distribution of allele frequencies (Figure S2A). As expected from binomial sampling (Figure S2B), for Pool-Seq datasets generated with no sequencing error, the number of SNPs remained always lower than the $\mathrm{AC}_{m>1 \%}$ datasets at the $1 \% \mathrm{MAF}$ threshold although increasing with coverages from $13.8 \%$ for PS30 ${ }_{m>1 \%}^{\epsilon=0}$ to $2.01 \%$ for PS200m $>1 \%{ }^{\epsilon=0}$ datasets (see Table S1 legend for details). Conversely, at the 5\% MAF threshold, the number of SNPs was slightly higher than the $\mathrm{AC}_{m>5 \%}$ datasets (from $2.58 \%$ for PS30 $0_{m>5 \%}^{\epsilon=0}$ to $1.51 \%$ for PS200 ${ }_{m>5 \%}^{\epsilon=0}$ ) which is related to i) the shape of the allele frequency spectrum (stochastic variation in read sampling leading to include more SNPs with $0.01<M A F<0.05$ than exclude SNPs with $M A F>0.05$ from the simulated genotying data because they are more numerous); and ii) variation in the simulated read coverages that explains the decreasing trend with $\lambda$. 
With sequencing errors, our filtering steps proved efficient to remove false SNPs except at the 1\% MAF threshold when $\epsilon=2.5 \%$ or when $\epsilon=1 \%$ at the lowest coverage $(\lambda=30$ and $\lambda=50)$. These configurations displayed substantial to very high proportions of false SNPs (up to $93.8 \%$ for $\operatorname{PS} 50_{m>1 \%}^{\epsilon=2.5 \%}$ ) although decreasing with coverage (Figure S1B). A 5\% MAF threshold always resulted in the complete removal of all the false SNPs for all the investigated scenarios (Table S1). Note that for the highest coverages, sequencing errors lead to a relative reduction of the number of SNPs due to the generation of spurious tri- or tetra- allelic SNPs from the simulated bi-allelic SNPs (compare e.g., PS200 $\underset{m>5 \%}{\epsilon=2.5 \%}$ and PS100 $0_{m>5 \%}^{\epsilon=2.5 \%}$ on Figure S1A).

\subsubsection{Comparison of poolfstat and Admixtools estimates for allele count data}

We first analyzed the 250 simulated $\mathrm{AC}_{m>1 \%}$ datasets to estimate with both poolfstat and Admixtools programs i) the 15 basis $f$-statistics (taking $P 1$ as the reference population) consisting of five $f_{2}$ and ten $f_{3}$ (Figure $3 \mathrm{~A}$ ) and their corresponding error covariance matrix (Figure 3B); ii) the $60 f_{3}^{\star}$ (Figure 3C) and their associated Z-scores (Figure 3D); and iii) the $45 \mathrm{D}$-statistics (Figure 3E) and their associated Z-scores (Figure 3F). The estimates were all found in almost perfect agreement between the two implementations with Mean Absolute Differences (MAD) negligible when compared to the range of variation of the underlying values. For $f-$ and $D$ - statistics, slight differences were mostly due to the plotted poolfstat estimates corresponded to block-jackknife means (i.e., excluding SNPs outside blocks as those from chromosome ends). Using poolfstat estimates based on all the SNPs indeed resulted in almost null MAD (MAD' in Figure 3A, C and E), up to rounding errors due to lower decimal precision in the printed output of the Admixtools programs. Note that the differences in block-jackknife implementation among the two programs (Material and Methods) had very minor impact on the estimation of error variance and covariance of the estimates (Figures 3B). Accordingly, the MAD computed on Z-scores remained very small (although inflated for higher values) and Z-score based decision for the underlying three-population admixture (Figures 3D) or four-population treeness tests (Figures 3F) were highly consistent (with a proportion $\beta=97.7 \%$ and $\beta=98.0 \%$ respectively of Z-scores significant with the two programs among the ones significant with at least one program).

\subsubsection{Performance of $f_{3}$ and $f_{3}^{\star}$ based tests of admixture and $f_{4}$ and $D$ - based tests of treeness for allele count and Pool-Seq data}

We ran the compute.fstats function on all the simulated allele count and Pool-Seq datasets to estimate all $f$ - and $D$ - statistics. To further evaluate the impact of (improperly) treating read counts as allele counts when 


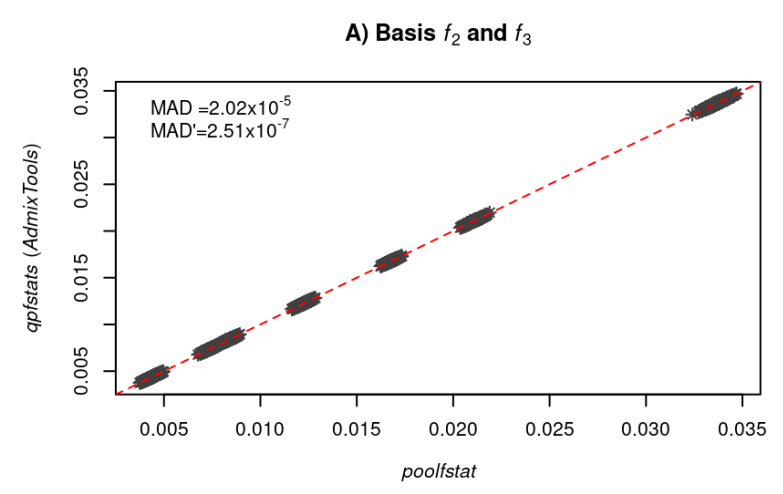

C) $f_{3}^{*}$

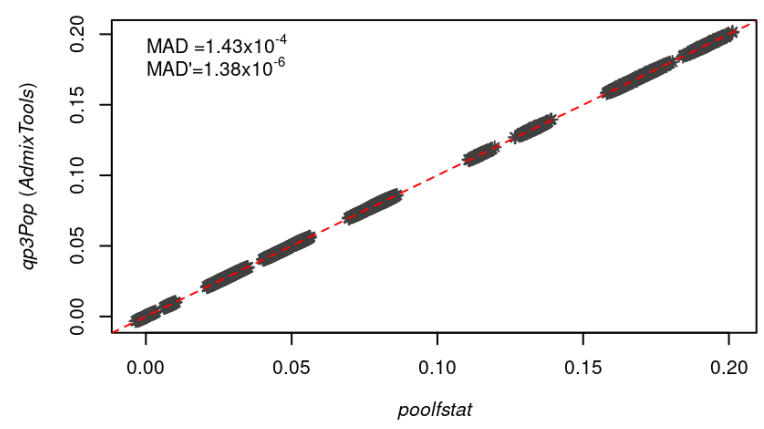

E) D-statistic

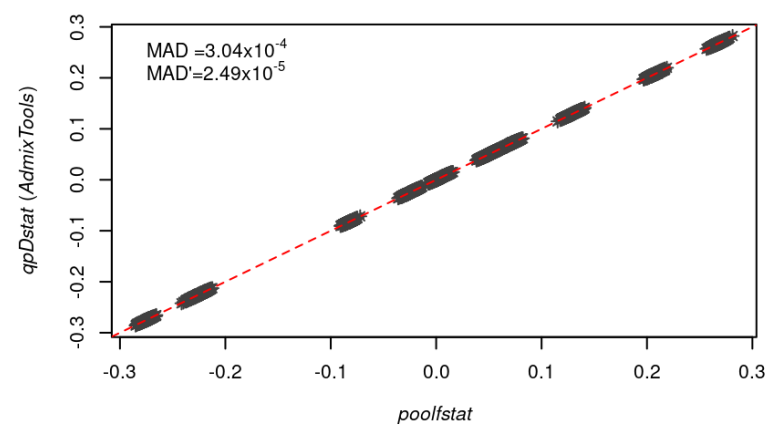

B) Error covariance matrix $(Q)$

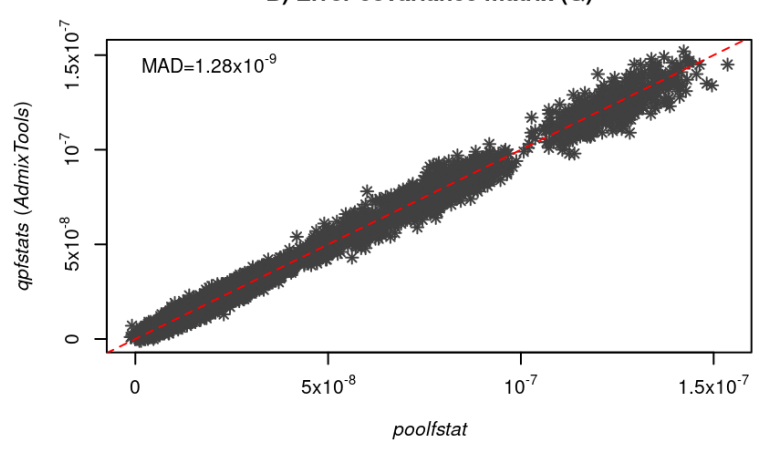

D) $f_{3}^{*}$ based Z-score

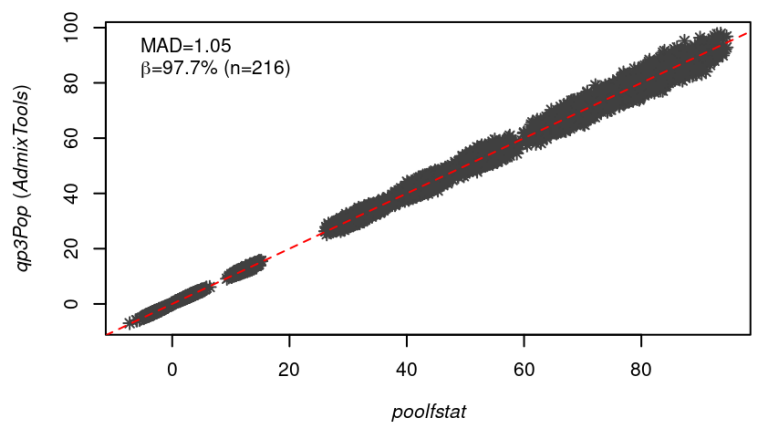

F) D-statistic based Z-score

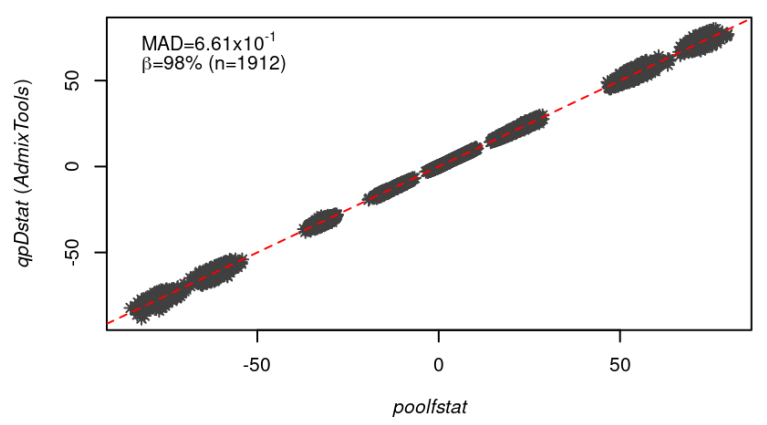

Figure 3. Comparison of poolfstat and AdmixTools estimates across 250 simulated allele count datasets $\left(\mathbf{A C}_{m \geq 1 \%}\right)$. A) All estimates of the 15 basis $f$-statistics taking $P 1$ as the reference population and corresponding to $5 f_{2}$ of the form $(P 1, P x)$ and the $10 f_{3}$ of the form $(P 1 ; P x, P y)$ (with $x=2, . ., 6 ; y=3, . ., 6$ and $\left.y>x\right)$. B) All Block-jackknife estimates of the covariance matrix $\boldsymbol{Q}$ of the 15 basis $f$-statistics (15 error variances and 105 error covariances). C) All estimates of the $60 f_{3}^{\star}$ (scaled $f_{3}$ ) and their associated Z-scores (D). E) All estimates of the $45 D$-statistics (scaled $f_{4}$ ) and their associated Z-scores (F). For each comparison, the Mean Absolute Difference (MAD) between the parameter estimates of the two programs are given on the upper left corner of the plots. In A), C) and E), poolfstat estimates correspond to block-jackknife means (i.e., they only include SNPs eligible for block-jackknife). The given MAD' value is the MAD between AdmixTools and poolfstat estimates that include all SNPs (see documentation for the compute. fstats function). In D), a consistency score $\beta$ is also given and was computed as the proportion of Z $\mathrm{Z}$-scores $<-1.65$ (i.e., significant three-population test of admixture at a $5 \%$ threshold) with both programs among the $n=216$ ones significant in at least one of the two programs. Similarly, in F), the given consistency score $\beta$ is computed as the proportion of absolute Z-scores $<1.96$ (i.e., passing the four-population treeness at a 5\% threshold) with both programs among the $n=1,912$ ones with an absolute Z-scores $<1.96$ in at least one of the two programs) 
analyzing Pool-Seq data we also analyzed the simulated Pool-Seq datasets (focusing only on PS $\lambda_{m>m_{t} \%}^{\epsilon=0}$ datasets, i.e., simulated without sequencing error) as if they were allele count data. Overall, 42 different configurations were thus investigated each originating from the 250 allele count datasets simulated under the demographic scenario represented in Figure 1, leading to a total of $42 \times 250=10,500$ analyses.

Tables 3 and S2 provide the estimated power (True Positive Rate, TPR) and False Positive Rate (FPR) of the $f_{3}$ - and $f_{3}^{\star}$-based test of admixture for each configurations. As P6 was the only admixed population, each TPR was estimated as the proportion of $f_{3}$ (respectively $f_{3}^{\star}$ ) with an associated Z-score $<-1.65$ (95\% significance threshold) for the (P6;P2,P3) population triplet (i.e., among 250 estimates). Conversely, the FPR was computed as the proportion of $f_{3}$ (respectively $f_{3}^{\star}$ ) with an associated Z-score $<-1.65$ among all the 50 population triplets that do not involve P6 as a target (i.e., among $12,250=250 \times 50$ estimates). Consistent with Patterson et al (2012), the performance of $f_{3}$ - and $f_{3}^{\star}$-based test of admixture were virtually the same for all the configurations. When the same MAF threshold was applied, the performance of Pool-Seq data generated with no sequencing error were very close to that obtained with allele count data although the power tended to slightly decrease with decreasing sequencing coverage. Interestingly, increasing the MAF threshold from $1 \%$ to 5\% increased the power by more than $10 \%$ and in all cases, no false positive signal of admixture was detected. Surprisingly, sequencing errors in Pool-Seq data also tended to increase the power from a negligible amount (less or close to 1\%) at 5\% MAF threshold to a quite substantial amount at 1\% MAF threshold (decreasing with coverage and increasing with sequencing error rate). At the extreme, a power of $100 \%$ was even observed when $\lambda \leq 50$ and $\epsilon \geq 1 \%$. This trend was actually directly related to the proportion of false SNPs introduced by sequencing error (Figure S1B) that resulted in a downward bias of $f_{3}$ and $f_{3}^{\star}$ estimates, although the underlying tests remained robust as all the estimated FPR were null except for PS50 ${ }_{m>1 \%}^{\epsilon=2.5 \%}$ data $(\mathrm{FPR}=6.47 \%)$ which displayed the highest proportion of false SNPs (> 90\%, Figure S1B). However, this observed apparent robustness of the three-population tests to false SNPs should be interpreted cautiously since it may rather result from the moderate to high expected $f_{3}$ and $f_{3}^{\star}$ values in our simulated scenario for the population triplets that do not involve P6 as a target. Overall, applying a 5\% MAF threshold on Pool-Seq data (even with $\epsilon=2.5 \%$ ) to remove false SNPs (see above) allowed recovering the performances similar to that obtained when analyzing datasets with no sequencing error. Finally, it is worth stressing that analyzing Pool-Seq data as allele counts, whatever the coverage or MAF threshold considered, lead to no power in detecting admixture event with $f_{3}$ or $f_{3}^{\star}$ based tests due to a strong upward estimation bias.

Tables 4 and S3 similarly provide the estimated power (TPR) and FPR of the $f_{4}$ - and $D$-based tests of treeness for the 42 configurations investigated in the simulation study. Given the simulated scenario, eight of the 45 differ- 


\begin{tabular}{|c|c|c|c|c|c|c|c|}
\hline \multirow{2}{*}{$\begin{array}{c}\text { MAF } \\
\text { threshold }\end{array}$} & seq. error & \multicolumn{5}{|c|}{ Pool-Seq (read counts) data } & \multirow{2}{*}{$\begin{array}{l}\text { allele count } \\
\text { data }\end{array}$} \\
\hline & $\epsilon$ & $\lambda=30$ & $\lambda=50$ & $\lambda=75$ & $\lambda=100$ & $\lambda=200$ & \\
\hline \multirow{4}{*}{$>1 \%$} & 0 & $82.0(0.00)$ & $84.4(0.00)$ & $86.0(0.00)$ & $86.0(0.00)$ & $85.2(0.00)$ & \multirow{4}{*}{$85.6(0.00)$} \\
\hline & $1 \% 0$ & $100(0.00)$ & $100(0.00)$ & $86.8(0.00)$ & $87.2(0.00)$ & $86.4(0.00)$ & \\
\hline & $2.5 \%$ & $100(0.00)$ & $100(6.47)$ & $99.6(0.00)$ & $92.8(0.00)$ & $88.4(0.00)$ & \\
\hline & 0 & $0.00(0.00)^{\star}$ & $0.00(0.00)^{\star}$ & $0.00(0.00)^{\star}$ & $0.00(0.00)^{\star}$ & $0.00(0.00)^{\star}$ & \\
\hline \multirow{4}{*}{$>5 \%$} & 0 & $93.6(0.00)$ & $95.2(0.00)$ & $96.4(0.00)$ & $96.0(0.00)$ & $96.0(0.00)$ & \multirow{4}{*}{$96.8(0.00)$} \\
\hline & $1 \% 0$ & $94.0(0.00)$ & $96.8(0.00)$ & $96.4(0.00)$ & $97.2(0.00)$ & $96.8(0.00)$ & \\
\hline & $2.5 \%$ & $94.0(0.00)$ & $96.0(0.00)$ & $96.0(0.00)$ & $97.2(0.00)$ & $96.8(0.00)$ & \\
\hline & 0 & $0.00(0.00)^{\star}$ & $0.00(0.00)^{\star}$ & $0.00(0.00)^{\star}$ & $0.00(0.00)^{\star}$ & $0.00(0.00)^{\star}$ & \\
\hline
\end{tabular}

Table 3. Comparison of the performance of $f_{3}$-based tests of admixture for different types of data simulated under the Figure 1 scenario processing poolfstat analyses. For each MAF threshold (MAF>1\% or MAF> 5\%), the table gives True and False (in parenthesis) Positive Rates (in \%) for 21 different types of analyses relying on i) allele count data; ii) 15 different Pool-Seq read count data (five mean coverages $\lambda$ and three sequencing error rates $\epsilon$ ); and iii) Pool-Seq read count data simulated with $\epsilon=0$ treated as allele counts (corresponding results of this bad practice are highlighted in italics and ${ }^{\star}$ ). Each TPR was computed from the analysis of 250 independent datasets (generated from the data simulated under Figure 1 demographic scenario) as the proportion of $f_{3}$ with an associated Z-score $<-1.65$ (95\% significance threshold) for the (P6;P2,P3) population triplet ( $\mathrm{n}=250$ estimates). The FPR was similarly computed as the proportion of $f_{3}$ with an associated $\mathrm{Z}$-score $<-1.65$ among all the 50 population triplets that do not involve $\mathrm{P} 6$ as target population ( $\mathrm{n}=250 \times 50=12,250$ estimates).

ent population quadruplets (namely (P1,P2;P3,P4); (P1,P2;P3,P5); (P1,P2;P4,P5); (P1,P3;P4,P5); (P1,P6;P4,P5); (P2,P3;P4,P5); (P2,P6;P4,P5); and (P3,P6;P4,P5)) have a null expected $F_{4}$ (and $D$ ) value. Note that this may easily be shown with the symbolic calculus derivation implemented in graph.params2symbolic.fstats (Table 2). For each configuration, the TPR of the treeness test was then estimated as the proportion of $f_{4}$ (respectively $D$ ) with an associated absolute Z-score $<1.96$ (95\% significance threshold) for these eight population quadruplets ((P1,P2;P3,P4); (P1,P2;P3,P5); (P1,P2;P4,P5); (P1,P3;P4,P5); (P1,P6;P4,P5); (P2,P3;P4,P5); (P2,P6;P4,P5); (P3,P6;P4,P5)) over all the 250 different underlying analyses (i.e., among 2,000 $=250 \times 8$ estimates). Conversely, the FPR was estimated as the proportion of $f_{4}$ (respectively $D$ ) with an associated absolute Z-score $<1.96$ among all the 37 remaining population quadruplets (i.e., among $9,250=250 \times 37$ estimates). The power for both $F_{4}$ - and $D$-based tests were remarkably consistent across all the different configurations. In addition, the tests were all found almost perfectly calibrated since the estimated power were close to $95 \%$, the probability of rejecting the null hypothesis at the chosen $95 \%$ significance threshold for Z-scores. Likewise, all FPR remained low $(\leq 0.15 \%)$, although increasing with MAF thresholds (more than twice higher for a given type of data when increasing the MAF threshold from $1 \%$ to 5\%). Overall, sequencing errors and coverage had no impact on the performance of the $f_{4}$ - and $D$-based test of treeness. As expected, analyzing read counts as allele count data did not affect the performance of these tests (see Discussion). 


\begin{tabular}{|c|c|c|c|c|c|c|c|}
\hline \multirow{2}{*}{$\begin{array}{c}\text { MAF } \\
\text { threshold }\end{array}$} & \multirow{2}{*}{$\begin{array}{c}\text { seq. error } \\
\epsilon\end{array}$} & \multicolumn{5}{|c|}{ Pool-Seq (read counts) data } & \multirow{2}{*}{$\begin{array}{l}\text { allele count } \\
\text { data }\end{array}$} \\
\hline & & $\lambda=30$ & $\lambda=50$ & $\lambda=75$ & $\lambda=100$ & $\lambda=200$ & \\
\hline \multirow{4}{*}{$>1 \%$} & 0 & $94.0(0.05)$ & $94.4(0.06)$ & $94.1(0.04)$ & $94.5(0.05)$ & $94.3(0.02)$ & \multirow{4}{*}{$94.2(0.02)$} \\
\hline & $1 \% 0$ & $94.3(0.04)$ & $94.2(0.03)$ & $94.3(0.03)$ & $94.3(0.03)$ & $94.1(0.05)$ & \\
\hline & $2.5 \%$ & $94.8(0.06)$ & $94.5(0.05)$ & $94.8(0.03)$ & $94.5(0.06)$ & $94.3(0.04)$ & \\
\hline & 0 & $94.0(0.05)^{\star}$ & $94.4(0.06)^{\star}$ & $94.1(0.04)^{\star}$ & $94.5(0.05)^{\star}$ & $94.3(0.02)^{\star}$ & \\
\hline \multirow{4}{*}{$>5 \%$} & 0 & $94.5(0.14)$ & $94.3(0.11)$ & $94.1(0.14)$ & $94.9(0.09)$ & $94.3(0.08)$ & \multirow{4}{*}{$94.3(0.11)$} \\
\hline & $1 \% 0$ & $94.5(0.09)$ & $94.5(0.11)$ & $94.5(0.13)$ & $94.2(0.09)$ & $94.1(0.15)$ & \\
\hline & $2.5 \%$ & $95.2(0.13)$ & $93.8(0.11)$ & $94.2(0.12)$ & $94.5(0.11)$ & $94.3(0.13)$ & \\
\hline & 0 & $94.5(0.14)^{\star}$ & $94.3(0.11)^{\star}$ & $94.1(0.14)^{\star}$ & $94.9(0.09)^{\star}$ & $94.3(0.08)^{\star}$ & \\
\hline
\end{tabular}

Table 4. Comparison of the performance of $f_{4}$-based test of treeness for different types of data simulated under the Figure 1 scenario processing poolfstat analyses. For each MAF threshold (MAF>1\% or MAF> 5\%), the table gives True and False (in parenthesis) Positive Rates (in \%) for 21 different types of analyses relying on i) allele count data; ii) 15 different Pool-Seq read count data (five mean coverages $\lambda$ and three sequencing error rates $\epsilon$ ); and iii) Pool-Seq read count data simulated with $\epsilon=0$ treated as allele counts (corresponding results of this bad practice are highlighted in italics and ${ }^{\star}$ ). Each TPR was computed from the analysis of 250 independent datasets (generated from the data simulated under Figure 1 demographic scenario) as the proportion of $f_{4}$ with an associated absolute Z-score $<1.96$ (95\% significance threshold) among all the eight population quadruplets ((P1,P2;P3,P4); (P1,P2;P3,P5); (P1,P2;P4,P5); (P1,P3;P4,P5); (P1,P6;P4,P5); (P2,P3;P4,P5); (P2,P6;P4,P5); (P3,P6;P4,P5)) with a null expected $F_{4}(\mathrm{n}=250 \times 8=2,000$ estimates). The FPR was similarly computed as the proportion of $f_{4}$ with an associated absolute Z-score $<1.96$ among all the 37 remaining population quadruplets $(\mathrm{n}=250 \times 37=9,250$ estimates).

\subsubsection{Precision of the $F_{4}$-ratio based estimation of the admixture rate $\alpha$}

Given the simulated scenario, two different ratios of $f_{4}$ estimates could be used to estimate the admixture proportion $\alpha=0.25$ (Figure 1), namely $\hat{\alpha}_{1}=\frac{f_{4}(P 1, P 4 ; P 3, P 6)}{f_{4}(P 1, P 4 ; P 2, P 3)}$ and $\hat{\alpha}_{2}=\frac{f_{4}(P 1, P 5 ; P 3, P 6)}{f_{4}(P 1, P 5 ; P 2, P 3)}$ (Patterson et al, 2012). The graph. params2symbolic. fstats function (Table 2) may also prove useful to identify appropriate quadruplets (Supplementary Vignette V2). We used the compute. f4ratio function to obtain these two estimates from all the simulated datasets together with their $95 \%$ CI (defined as $\hat{\alpha} \pm 1.96 \hat{\sigma}_{\alpha}$ where $\hat{\sigma}_{\alpha}$ is the block-jackknife standarderror estimate). Tables 5 and S4 provide the mean of the estimated $\hat{\alpha}_{1}$ and $\hat{\alpha}_{2}$ respectively over the 250 analyzed datasets for each of the 42 investigated configurations. As expected from the above evaluation of treeness tests, estimates of $\alpha$ were highly consistent among all the investigated configurations and similar for the two considered $f_{4}$-ratio with a mean value varying between 0.245 and 0.248 . Yet, a slight downward bias (always $<2 \%$ ) could be noticed but the estimated $95 \%$ CIs were almost always optimal (or close to) since they contained the true simulated value ( $\alpha=0.25$ ) from $90.0 \%$ to $95.2 \%$ of the time (Tables 5 and S4).

\subsubsection{Evaluation of graph fitting}

We further estimated for all the simulated datasets branch lengths in drift units and admixture proportion $\alpha$ with their $95 \%$ CIs by fitting the simulated graph with fit.graph. As for the $f_{4}$-ratio based estimation, estimates of 


\begin{tabular}{|c|c|c|c|c|c|c|c|}
\hline \multirow{2}{*}{$\begin{array}{c}\text { MAF } \\
\text { threshold }\end{array}$} & \multirow{2}{*}{$\begin{array}{c}\text { seq. error } \\
\epsilon\end{array}$} & \multicolumn{5}{|c|}{ Pool-Seq (read counts) data } & \multirow{2}{*}{$\begin{array}{c}\text { allele count } \\
\text { data }\end{array}$} \\
\hline & & $\lambda=30$ & $\lambda=50$ & $\lambda=75$ & $\lambda=100$ & $\lambda=200$ & \\
\hline \multirow{4}{*}{$>1 \%$} & 0 & $0.247(92.4)$ & $0.247(92.8)$ & $0.247(94.4)$ & $0.247(93.6)$ & $0.247(92.0)$ & \multirow{4}{*}{$0.247(92.8)$} \\
\hline & $1 \% 0$ & 0.248 (91.6) & $0.247(91.6)$ & $0.247(92.4)$ & $0.247(93.2)$ & $0.247(92.0)$ & \\
\hline & $2.5 \%$ & $0.247(91.6)$ & $0.246(94.0)$ & $0.248(93.2)$ & $0.247(91.2)$ & $0.248(92.0)$ & \\
\hline & 0 & $0.247(92.4)$ & $0.247(92.8)^{\star}$ & $0.247(94.4)^{\star}$ & $0.247(93.6)^{\star}$ & $0.247(92.0)^{\star}$ & \\
\hline \multirow{4}{*}{$>5 \%$} & 0 & $0.247(92.4)$ & $0.248(93.2)$ & 0.247 (93.6) & $0.247(93.2)$ & $0.247(92.4)$ & \multirow{4}{*}{$0.247(92.4)$} \\
\hline & $1 \% 0$ & $0.248(93.2)$ & $0.247(91.6)$ & $0.247(91.6)$ & $0.247(92.8)$ & $0.247(91.2)$ & \\
\hline & $2.5 \%$ & $0.247(91.6)$ & $0.246(95.2)$ & 0.248 (93.2) & $0.247(90.0)$ & $0.248(93.2)$ & \\
\hline & 0 & $0.247(92.4)^{\star}$ & $0.248(93.2)^{\star}$ & $0.247(93.6)^{\star}$ & $0.247(93.2)^{\star}$ & $0.247(92.4)^{\star}$ & \\
\hline
\end{tabular}

Table 5. Comparison of $F_{4}$-ratio based estimation of the simulated admixture proportion $\alpha$ in Figure 1 scenario for different types of data processing poolfstat analyses. For each MAF threshold (MAF> $1 \%$ or MAF> 5\%), the table gives the mean of the estimated $\hat{\alpha}=\frac{f_{4}(P 1, P 4 ; P 3, P 6)}{\left.f_{4}(P 1 P 4) \cdot P 2, P 3\right)}$ (across 250 independent simulated datasets) for 21 different types of analyses relying on i) allele count data; ii) 15 different Pool-Seq read count data (five mean coverages $\lambda$ and three sequencing error rates $\epsilon$ ); and iii) Pool-Seq read count data simulated with $\epsilon=0$ treated as allele counts (corresponding results of this bad practice are highlighted in italics and ${ }^{\star}$ ). The proportion (in \%) of the 250 estimated $95 \%$ CIs that contain the true simulated value $(\alpha=0.25)$ is given in parenthesis.

$\alpha$ were virtually unbiased and consistent across all the 42 different investigated configurations (Figure S3). Nevertheless, the $95 \%$ CIs were always too narrow since they contained the actual value ( $\alpha=0.25$ ) from only $40.8 \%$ to $74.4 \%$ of the time (Table S5) as expected from the $\chi^{2}$ approximation of the LRT underlying the computation of these CIs. Figures 4 and S4 plot the distributions of the estimated lengths for the ten branches of the simulated admixture graph branches (over the 250 estimates per configuration) when applying 5\% and $1 \%$ MAF threshold respectively. The corresponding mean estimates and proportions of 95\% CI's including the true value are provided in Tables S6 to S15. Note that the branches $P 8 \leftrightarrow R$ and $P 9 \leftrightarrow R$ that are connected to the root $R$ (Figure 1) can only be estimated jointly (as $\tau_{P 8 \leftrightarrow P 9}=\tau_{P 8 \leftrightarrow R}+\tau_{P 9 \leftrightarrow R}, R$ being arbitrarily set in its middle).

At the 5\% MAF threshold, very similar performance were obtained for the allele count and the different PoolSeq datasets whatever the simulated read coverage or sequencing error rates (Figures 4A, 4B, and 4C). Hence, mostly unbiased branch lengths were estimated for the four leaves (terminal branches) $\tau_{P 1 \leftrightarrow P 7}, \tau_{P 2 \leftrightarrow S 1}, \tau_{P 6 \leftrightarrow S}$ and $\tau_{P 3 \leftrightarrow S 2}$. As previously observed with $\alpha$, the estimated $95 \%$ CI's remained too narrow particularly for $\tau_{P 2 \leftrightarrow S 1}$ for which less than $50 \%$ of the CI's contained the true value (Table S7) compared to more than $80 \%$ for $\tau_{P 1 \leftrightarrow P 7}$ (Table S6). As expected from the the drift-scaling approximation, the estimated branch lengths tended to be slightly downwardly biased (ca. $2 \%)$ for the two other leaves $\left(\tau_{P 4 \leftrightarrow P 9}\right.$ and $\left.\tau_{P 5 \leftrightarrow P 9}\right)$ but the estimated $95 \%$ CI displayed similar characteristics since from $48.0 \%$ to $87.6 \%$ contained the true values, the proportion increasing in Pool-Seq datasets when coverage and sequencing error decreased (Tables S10 and S11). Conversely, the internal branch lengths tended to be upwardly biased from a slight (ca. 2\%) for $\tau_{S 1 \leftrightarrow P 7}, \tau_{P 7 \leftrightarrow P 8}$ and $\tau_{S 2 \leftrightarrow P 8}$ (Tables S12 
to S14), to a moderate amount (ca. 20\%) for the root including branch $\tau_{P 8 \leftrightarrow P 9}$, the true value being then always outside the estimated 95\% CI's (Table S15). Yet, when analyzing data with a lower MAF threshold of 1\%, this bias almost completely vanished (Figure S4 and Table S15).

For the other branches, the estimates had similar characteristics (yet with a slightly decreased performance for the $\tau_{P 4 \leftrightarrow P 9}$ and $\tau_{P 5 \leftrightarrow P 9}$ leaves) for allele count data or Pool-Seq data simulated without sequencing error (Figure S4A). In agreement with previous observations, at the $1 \%$ MAF threshold, sequencing errors lead to strong downward bias at the lowest simulated coverages, i.e., when the percentage of false SNPs became non-negligible (Figures S4B and S4C). Finally, whatever the chosen MAF threshold, improperly analyzing read counts as allele count data always lead to a substantial upward bias of the lengths of all the leaves (Figure 4D). Notice however, that this had no or limited impact on the estimation of internal branch lengths.

\subsubsection{Evaluation of graph construction}

To provide insights into the reliability of graph construction, we evaluated the performance of the add.leaf function in positioning the admixed population P6 on the underlying (((P1,P2),P3),(P4,P5)) tree (Figure 1) for the different types of simulated datasets. Table S16 gives the proportion of correctly inferred admixture graphs (i.e., corresponding to the simulated scenario) with a $\Delta_{B I C}>6$ support with all other tested graphs over the 250 analyzed datasets for each of the 42 investigated configurations. As the reference tree with rooted topology $(((\mathrm{P} 1, \mathrm{P} 2), \mathrm{P} 3),(\mathrm{P} 4, \mathrm{P} 5))$ consists of eight branches, $\mathrm{P} 6$ may be connected with either i) nine non-admixed edges (connection to either one of the eight branches or as an outgroup) or; ii) $\left(\begin{array}{l}8 \\ 2\end{array}\right)-1=27$ admixed edges from two-way admixture events. Except for the PS50 ${ }_{m>1 \%}^{\epsilon=2.5 \%}$ dataset (the one with the highest percentage of false SNPs), the correct admixture graph was always retrieved with a fairly high support $\left(\Delta_{B I C}>15\right)$.

\subsection{Analysis of real Drosophila suzukii Pool-Seq data}

We here sketched the main findings from the analyses using poolfstat of a subset of the Pool-Seq data previously generated by Olazcuaga et al (2020) focusing on 14 population samples of the invasive species $D$. suzukii. For more details, we encourage readers to consult the Supplementary Vignette V2.

\subsubsection{Structuring of genetic diversity across the 14 populations}

Overall, the estimated global $F_{S T}$ across the 14 populations was $7.03 \%$ (95\% CI; [6.90\%; 7.32\%]). Estimates of all the pairwise-population $F_{S T}$ confirmed that populations tended to cluster according to their geographic area of 
A) PS with $\varepsilon=0$ (MAF>5\%)

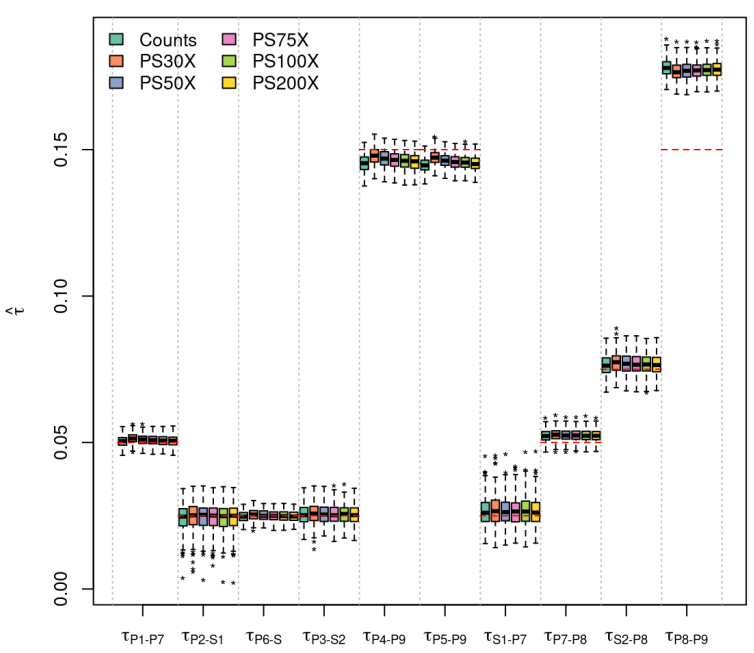

C) PS with $\varepsilon=0.25 \%(\mathrm{MAF}>5 \%)$

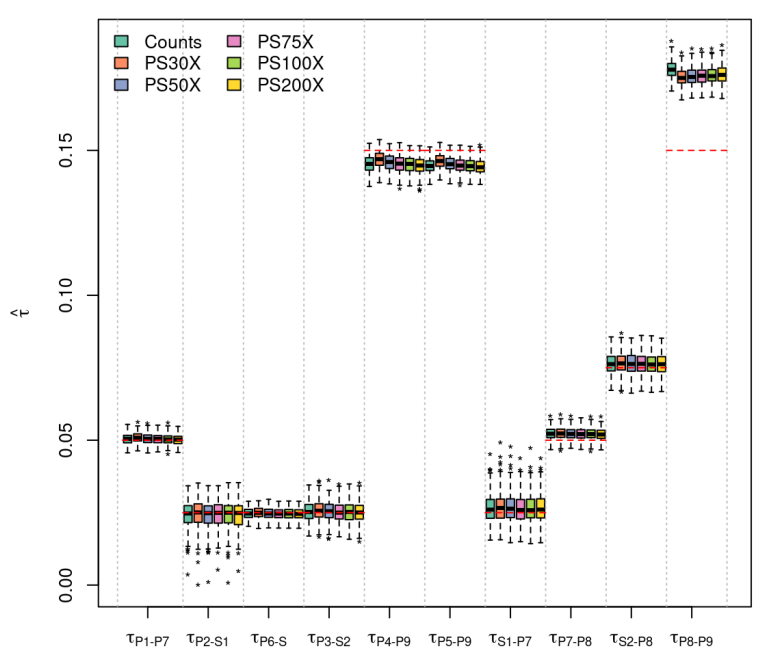

B) PS with $\varepsilon=0.1 \%($ MAF $>5 \%)$

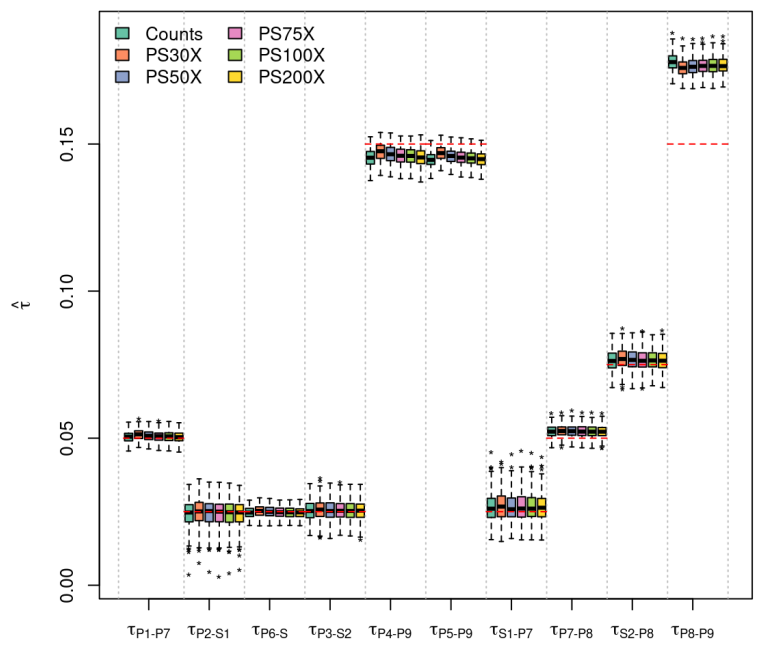

D) PS with $\varepsilon=0$ analyzed as allele counts (MAF $>5 \%$ )

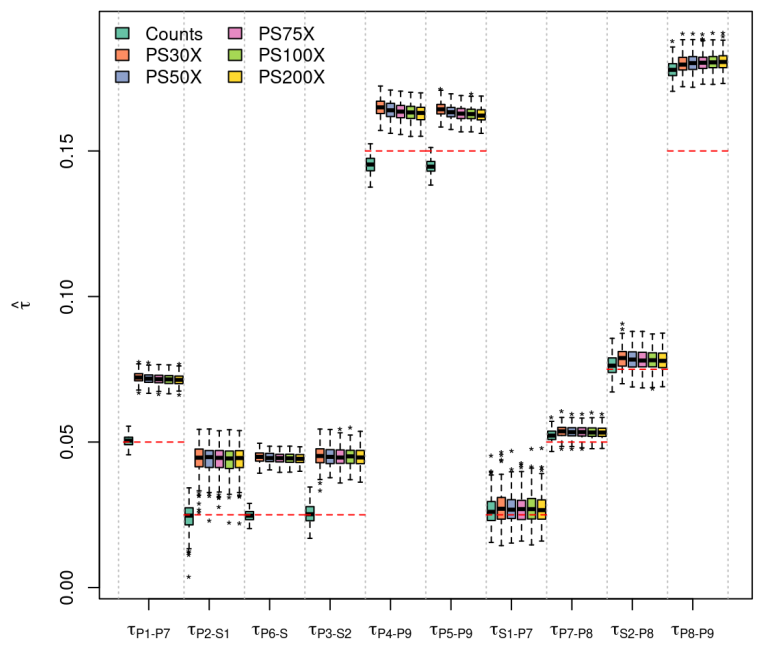

Figure 4. Distribution of the estimated drift-scaled lengths for all the branches in Figure 1 simulated scenario using admixture graph fitting (as implemented in the fit. graph function of poolfstat) for different types of data with a 5\% threshold on the overall SNP MAF. Each box plot summarize the distribution of the 250 estimated lengths of each of the ten branches obtained from the analysis of either allele count dataset ("Counts") or one of the five different simulated Pool-Seq read count datasets ("PS $\lambda X ")$ with different mean coverages $(\lambda=30 ; 50 ; 75 ; 100$; and200) as generated from the genotyping data simulated under the scenario depicted in Figure 1. Pool-Seq read count data were generated with no sequencing errors $(\epsilon=0)$ in A) and D) and with a sequencing error rate of $\epsilon=1 \% 0$ and $\epsilon=2.5 \%$ in panel B) and C) respectively (Table S1). In D), the read count data were analyzed as allele counts which corresponds to a bad practice. Note that the two branches coming from the root are combined since the position of the root is not identifiable by the model (i.e., $\tau_{P 8 \leftrightarrow P 9}=\tau_{P 8 \leftrightarrow R}+\tau_{P 9 \leftrightarrow R}$ ). Note that the box plots obtained from the analysis of count data are replicated in each panel for comparison purposes. For each branch, a red dotted line indicates the underlying simulated value. For Pool-Seq data, the overall MAF was estimated from read counts. 
origin (i.e., Asia, America and Hawaii; Figure 2A), with some geographically close populations showing low level of differentiation. For instance, in the American invasive area the US-Nca, US-Col and US-Nca populations all displayed pairwise $F_{S T}$ significantly lower than $1 \%$. Likewise, in the native area, the three populations CN-Bei, $\mathrm{CN}-\mathrm{Nin}$ and $\mathrm{CN}-\mathrm{Lia}$ originating from North-Western China were all found very closely related (all pairwise $F_{S T}$ being lower or very close to $1 \%$ ). Conversely, the Hawaiian sample (US-Haw) was found the most highly differentiated with all the other populations, all pairwise $F_{S T}$ including US-Haw ranging from $11.7 \%$ (with US-Sok) to $17.0 \%$ (with US-Col) suggesting strong drift in this population as confirmed by its lowest estimated heterozygosity (Supplementary Vignette V2).

\subsection{2 $f_{3}$-based tests of admixture suggest pervasive admixture in the invaded area}

Out of the 14 sampled populations, two (CN-Lia and JP-Tok) from the Asian native area and four (US-Col, USNca, US-Wat and US-Wis) from the continental American invasive areas showed at least one significantly negative $f_{3}$ at the $95 \%$ significance threshold (i.e., Z-score $<-1.65$ ). Table 6 summarizes for each of these 6 populations the number of significantly negative $\mathrm{f} 3$ together with the triplet with the lowest Z-score giving insights into the pair of populations that branch the closest to the two original sources (assuming a two-way admixture event). Except for CN-Lia, all the detected signals were significant at a far more stringent threshold (e.g., Z-score $<-2.33$ at $99 \%$ significance threshold). The $f_{3}$ and $f_{3}^{\star}$ statistics gave almost exactly the same results (Supplementary Vignette V2).

\begin{tabular}{|l|l|c|c|}
\hline Population & Origin & $\begin{array}{c}\text { nb. of signif. } \\
\text { tests }(\mathrm{f} 3 Z<-1.65)\end{array}$ & $\begin{array}{c}\text { triplet with the lowest } \\
\text { f3 Z-score }\end{array}$ \\
\hline CN-Lia & Native & 1 & CN-Lia;CN-Shi,JP-Sap (Z=-1.66) \\
\hline JP-Tok & Native & 11 & JP-Tok;CN-Nin,JP-Sap (Z=-7.11) \\
\hline US-Col & Invasive (AM) & 2 & US-Col;BR-Pal,US-Wis (Z=-3.31) \\
\hline US-Nca & Invasive (AM) & 6 & US-Nca;JP-Sap,US-Col (Z=-3.89) \\
\hline US-Wat & Invasive (AM) & 13 & US-Wat;US-Sdi,US-Sok (Z=-23.6) \\
\hline US-Wis & Invasive (AM) & 4 & US-Wis;JP-Sap,US-Col (Z=-5.02) \\
\hline
\end{tabular}

Table 6. Results of the $f_{3}$-based tests of admixture on populations from the $\mathbf{D}$. suzukii invasive species.For all the population displaying at least one significant signal of admixture at the $95 \%$ significance threshold (f3 $Z<-1.65$ ), the table gives the number of significant tests (out of the $C_{2}^{13}=78$ performed per population) and the triplet displaying the lowest Z-score (i.e., most significant test).

In the native area, JP-Tok showed clear evidence of admixture with 11 significant tests that all involved JP-Sap (from Northern Japan) as a source proxy. The three lowest $f_{3}$ values were obtained with three Chinese populations (CN-Nin, CN-Bei and CN-Shi in increasing order of $f_{3}$ ). Assuming an admixture-graph like history, this suggests 
that the two populations branching the closest to the two sources of JP-Tok were JP-Sap and CN-Nin. The remaining Chinese population, $\mathrm{CN}-\mathrm{Lia}$ showed some weak evidence for admixture with only one test barely significant at the $95 \%$ threshold for the triplet involving CN-Shi and JP-Sap as source proxies (Table 6).

Out of the seven invasive populations from continental America, the four populations US-Col, US-Wis, US-Nca and US-Wat showed strong evidence of admixture. Although it has up to now been considered as the closest to the first invading population of Continental America (based on historical records), the Western American US-Wat population displayed the strongest signals with 11 (strongly) significant tests. Interestingly, the three signals supported by the lowest (and hence more significant) Z-score all involved pairs of source population proxies originating from the continental American invasive area namely, in order of increasing Z-score (i.e., decreasing evidence), the (USSdi,US-Sok); (BR-Pal,US-Sok) and (US-Col,US-Sok) pairs. As the underlying $f_{3}$ CI's did not overlap with those of the other triplet configurations, these three pairs of populations may be considered as the closest (among the sampled populations) to the original US-Wat source populations. It is worth noting that the Western American US-Sok population was involved in nine of the 11 significant negative $f_{3}$ statistic with US-Wat as a target. The three others populations, US-Col, US-Wis and US-Nca only had a moderate number of significant tests (compared to others). Such tests always involved at least one of the two other populations and overlapping $f_{3}$ CI's. This suggests complex patterns of recurrent admixture event among US-Col, US-Wis and US-Nca, a feature consistent with their low level of differentiation and close geographic origins.

\subsubsection{Exploring invasion scenarios with admixture graph construction and fitting}

To provide further insights into the relationships of the surveyed populations and the probable scenarios of invasion of D. suzukii in the American area, we relied on admixture graph construction. Our purpose was not to build a comprehensive admixture graph for the 14 populations, which may be elusive given the close relationships of some populations and the pervasiveness of recent and presumably recurrent admixture events among the different populations, but rather to identify key regional event that occurred at early time of the invasion history of the species. From our extensive analyses (Supplementary Vignette V2), we were in fine able to build and estimate the parameters of two admixture graphs represented in Figure 2B and C. The first admixture graph described the somewhat complex and so far non-investigated relationships among the populations of the native area (including the early invasive population established in Hawaii since 1980) with a very good fit since the Z-score of the residuals for the worst fitted $f$-statistics was 1.06 (Figure 2B). In agreement with previous findings (and geographic proximity), the Hawaiian population was found more closely related to the Japanese population JP-Sap than to the 
other Chinese populations but it experienced a strong differentiation from their common ancestor (named JP in Figure 2B) with an estimated branch length of 0.255 drift units $\left(\frac{t}{2 N_{e}}\right)$. Yet, it was not possible from our data to definitively conclude that US-Haw originates from a Japanese population since we have no element to claim that the (ancestral) node population JP was located in Japan. To that end additional sampling of Japanese populations would be required. The inferred graph also confirmed above $f_{3}$-based test results of an admixed origins of JP-Tok between a population closely related to JP-Sap (the main contributor) and a second source likely of Chinese origin although the same caution as for JP are needed regarding the geographic origins of this internal node populations. Similarly, CN-Lia was found admixed with a contributing source of Chinese ancestry related to CN-Shi largely predominant (estimated contribution $\widehat{\alpha}_{C}=96.0 \% ; 95 \%$ CI, [95.7;96.3]), and a second (minor) contributing source of presumably Japanese origin (related to JP-Sap). This may explain why the corresponding $f_{3}$-based test was only barely significant (Table 6). Interestingly, the graph topology also allowed estimating the Chinese ancestry of CN-Lia based on $F_{4}$-ratio resulting in consistent but larger 95\% CI ( $\widehat{\alpha}_{C}=95.6 ; 95 \%$ CI, [94.4;96.8]) as expected from above simulation study. CN-Nin, the remaining population from the native area, could not be positioned with reasonable accuracy onto the admixture graph of Figure $2 \mathrm{~B}$, the resulting worst fitted $f$-statistics associated to the best fitting graph having a Z-score=3.43. However, both its genetic proximity with CN-Lia and the best fitting admixture graph resulting from its positioning onto the scaffold tree including US-Haw, JP-Sap, CN-Bei and CNShi suggested a small amount of Japanese introgression (see Supplementary Vignette V2 for more details).

The second admixture graph represented in Figure $2 \mathrm{C}$ allowed providing insights into the history of introduction of $D$. suzukii into the American continent. It related the three continental American population, US-Sok, US-Wat and BR-Pal to a scaffold including the four unadmixed populations US-Haw, JP-Sap, CN-Shi and CN-Bei with a good fit (the worst fitted $f$-statistics had a Z-score=-1.83). The underlying scenario suggested that continental American populations originated from at least two major and successive admixture events. The first admixture event lead to the internal node population named Am1 and occured in balanced proportions between two sources, a Japanese one closely related to JP-Sap and a Hawaiian one relatively distantly related to US-Haw (according to the estimated branch lengths). The US-Sok population was the sampled continental American population the closest to Am1 and may thus be assumed the most closely related to the first invading population (in agreement with $f_{3}$-based test results). Yet US-Sok remained separated by about 0.0816 drift units from Am1 which may explain why no significantly negative $f_{3}$ were found for triplets with US-Sok as a target.

The second major admixture events occurred between the internal node population named Am2 and a Chinese population closely related to the common ancestor of CN-Bei and CN-Shi, with CN-Shi contributing slightly 
more (58.5\% against $41.5 \%$ for the other Am1 related ancestor). Interestingly, the closest Am2 representatives among the sampled populations were BR-Pal and US-Sdi (also in agreement with $f_{3}$-based tests) suggesting a more Southern geographical origin for Am2. We found that some additional ancestry from a ghost population or recurrent admixture events (e.g., related to Hawaiian populations) may also have contributed to US-Sdi, but this lead to a poor fit (worst fitted $f$-statistics Z-score $=-5.87$ for the best fitting graph resulting from the positioning of US-Sdi onto the graph, see Supplementary Vignette V2). Therefore, US-Sdi is not represented in Figure 2C. Although geographically distant, the Brazilian population BR-Pal thus appeared as the best proxy for Am2 thereby suggesting a rapid spread of $D$. suzukii in South America from this population without any subsequent admixture events. Additional (and preferably ancient) sample from South-American populations would help refining this scenario. Finally, according to the inferred graph, US-Wat was found to originate from a recent admixture between a population very closely related to US-Sok (and thus Am1) and a population deriving from Am2 with similar contributions of both.

In agreement with $f_{3}$-based admixture tests that suggested complex admixture histories among the closely related US-Col, US-Wis and US-Nca populations, no satisfactory admixture graph could be found when trying to position each of these onto the Figure $2 \mathrm{C}$ graph. Nevertheless, their resulting best fitted graphs all suggested a high contribution of the Am2 admixed source, a second contributing source being related to Japanese populations (Supplementary Vignette V2).

\section{Discussion}

\subsection{A new version of poolfstat for $f$-statistics estimation and associated inference from both Pool-Seq and allele count data}

The R package poolfstat was originally developed by Hivert et al (2018) to implement an unbiased estimator of $F_{S T}$ for PoolSeq data and provide utilities to facilitate manipulation of such data. We here proposed a substantially improved version that implements unbiased estimators of $F_{2}, F_{3}$ and $F_{4}$ parameters together with their scaled versions (i.e., pairwise $F_{S T}, F_{3}^{\star}$ and $D$ respectively). Although we primarily focused on the analysis of Pool-Seq data, we extended the package to analyze standard allele count (as obtained from individual genotyping or sequencing data) and to implement unbiased estimators equivalent to those available in the AdmixTools suite (Patterson et al, 2012) allowing us in turn to validate our estimation procedure. Recently, the admixr package was 
developed to interface most of the AdmixTools programs with $\mathrm{R}$ for the estimation of $f$-statistics (only from allele count data), with the noticeable exception of the admixture graph fitting program qpGraph (Petr et al, 2019). We implemented in poolfstat our own functions for fitting, building, visualizing and quality assessment of admixture graphs based on the estimated $f$-statistics. The underlying procedures shared strong similarities with those implemented in qpGraph (Patterson et al, 2012) resulting on the same fitting on some examples (e.g., Supplementary Vignette V1) or also MixMapper (Lipson et al, 2013, 2014) programs. As recognized by the developers themselves, the latter program specifically developed for admixture inference from allele count data was written in $\mathrm{C}++$ and MATLAB making it 'cumbersome to use' for users, as ourselves, with no MATLAB license. Moreover, to facilitate local exploration of the admixture graphs space, we also implemented in poolfstat efficient semi-automated building utilities (add. leaf and graph.builder functions). It should be noticed that although it does not include functions for the estimation of $f$-statistics, the admixturegraph $\mathrm{R}$ package (Leppälä et al, 2017) also provides several alternative valuable utilities for the fitting (based on a slightly different approach), the manipulation, and the visualization of admixture graphs together with utilities for the plotting of the statistics with their confidence intervals or the symbolic derivation $f$-statistics (as poolfstat). Overall, our effort of developing with poolfstat a self-contained, efficient and user-friendly R package capable of performing the entire workflow for $f$-statistics based demographic inference from both standard allele count and Pool-Seq read count data will hopefully make such a powerful framework accessible to a wider range of researchers and biological models.

\subsection{A unified definition of the $F$ parameters in terms of probability of gene identities}

To derive our unbiased estimators, we proposed to recapitulate and unify the different definitions of the $F$ and $D$ parameters in terms of probability of gene identity within population $\left(Q_{1}\right)$ or between pairs of populations $\left(Q_{2}\right)$ as summarized in equation 1 . This formulation offers a complementary perspective to the original description of these parameters in terms of covariance of allele frequencies (Patterson et al, 2012). In practice, a little algebra shows that the unbiased estimators derived from these two alternative formulations are strictly equivalent (i.e., when comparing eq. 6 for allele count data with Appendix A in Patterson et al, 2012). Formally, the $Q_{1}$ and $Q_{2}$ probabilities can be viewed as expected identity (in state) of genes across independent replicates of the (stochastic) evolutionary process (Rousset, 2007) that may themselves be expressed as a function of other demo-genetic population parameters. Hence, the obtained expressions for $F_{2}, F_{3}$ and $F_{4}$ in terms of $Q_{1}$ and $Q_{2}$ probabilities can be directly related to those by Peter (2016) in terms of coalescent times which allowed him to provide an in-depth exploration of their theoretical properties under a wide range of demographic models other than admixture graphs (see e.g., Figure 7 in 
Peter, 2016). More precisely, under an infinite-site mutation model with constant per-generation mutation rate $\mu$, the probability that two genes are identical in state is $Q=\sum_{t=1}^{\infty} C_{t}(1-\mu)^{2 t}=1-2 \mu \mathbb{E}[T]+O\left(\mu^{2}\right)$, where $C_{t}$ is the probability that the two genes coalesced $t$ generations in the past and $\mathbb{E}[T] \equiv \sum_{t=1}^{\infty} t C_{t}$ is the expected coalescence time of two genes (see Slatkin, 1991; Rousset, 2007, pp.58-59). Using $Q_{1}^{(1)}=1-2 \mu \mathbb{E}\left[T_{11}\right]$ and $Q_{1}^{(2)}=1-2 \mu \mathbb{E}\left[T_{22}\right]$ as the IIS probabilities within populations 1 and 2 respectively and $Q_{2}=1-2 \mu \mathbb{E}\left[T_{12}\right]$ as the IIS probability between 1 and 2 allows recovering equations 16 (after fixing a typo into it), 20c and 24 by Peter (2016) for $F_{2}, F_{3}$ and $F_{4}$ respectively. Likewise, the estimators derived from (unbiased) estimators of $Q_{1}$ and $Q_{2}$ are equivalent to those expressed in terms of average pairwise differences between and within populations which are natural estimators for $2 \mu \mathbb{E}[T]$ terms as proposed by Peter (2016, eq. 17) for $F_{2}$ estimator based on allele count data (e.g., noting that $\hat{\pi}_{11}=1-\hat{Q}_{1}^{1}, \hat{\pi}_{22}=1-\hat{Q}_{1}^{2}$ and $\hat{\pi}_{12}=1-\hat{Q}_{2}$ following his notations). For Pool-Seq data, replacing the latter estimators of nucleotide diversities by the unbiased estimators described in Ferretti et al (2013, eqs. 3 and 10) would also result in the same estimator for $F_{2}$ (and other parameters) as those defined in our equation 6 .

In practice, estimators are obtained by averaging over (a high) number of SNPs which amounts assuming that each represent an independent outcome of a common demographic processes that shaped the genome-wide patterns of genetic diversity. This generally allows to provide accurate estimations and LD between markers (i.e., violation of the marker independence assumption) can be accounted for with block-jackknife estimation of standard errors (Patterson et al, 2012). Importantly, as originally noticed by Patterson et al (2012), expressions of $F_{2}, F_{3}$ and $F_{4}$ in terms of coalescent times (Peter, 2016) show explicitly that they both depend on the demography (via $\mathbb{E}[T]$ ) and the marker mutation rate $(\mu)$. In the scaled versions of $F_{2}$ and $F_{3}\left(F_{S T}\right.$ and $F_{3}^{\star}$ respectively), the parameter $\mu$ cancels out making them presumably more comparable across different datasets. It should however be noticed, that for demographic inference purposes, scaling of the $f$-statistics is not needed. Indeed, the three-population test of admixture is informed by the sign of $f_{3}$ which is not affected by the denominator of $F_{3}^{\star}$. Similarly, the fourpopulation test evaluates departure of $f_{4}$ (i.e., the numerator of $D$ ) from a null value expected under the hypothesis of treeness. Patterson et al (2012) also showed both analytically and using simulations that $F_{3}$ and $F_{4}$ estimates remained mostly robust to various realistic SNP ascertainment scheme. It is finally worth stressing that admixture graph inference only requires additivity of $F_{2}$ (Patterson et al, 2012), a feature not fulfilled by $F_{S T}$ (or $F_{3}^{\star}$ and $D$ ).

\subsection{Estimation of $f$-statistics and inference from Pool-Seq data}

Our simulation study showed that accurate estimates of $F$ and $D$ parameters could be obtained from Pool-Seq data from the unbiased estimators we developed, thereby extending our findings for the Pool-Seq $F_{S T}$ estimator (Hivert 
et al, 2018). With no sequencing error, this remained true even at a read coverage as low as $30 \mathrm{X}$ which was here lower than our simulated haploid sample size of 50. Increasing the coverage only provided marginal gain. When introducing sequencing errors, the performance of the estimators tended to decrease for the lowest investigated read coverages (up to 50X) and MAF filtering threshold. This was however essentially due to the presence of spurious SNPs that were not completely filtered out when considering too loose criteria. As a result, simply increasing the threshold on the overall MAF (computed from read counts over all the pool samples) to 5\% allowed to remove all the spurious SNPs and recover accurate estimates of the parameters at the lowest read coverages. In agreement with original observations made for allele count data (Patterson et al, 2012), all the $f$-statistics based analyses (i.e., three-population test of admixture, four-population test of treeness, $F_{4}$-ratio estimation of admixture proportions or admixture graph fitting) remained remarkably robust to a MAF-based ascertainment process. From our simulation study, discarding lowly polymorphic SNPs was only found to increase the bias of the drift-scaled length estimates of internal branch in admixture graph. In practice, cost-effective designs consisting of sequencing pools of 30 to 50 individuals at a 50-100X coverage and applying MAF threshold of 5\% to filter the called SNPs are expected to provide good performance for all the different $f$-statistics based inference methods we presented here.

For Pool-Seq data, all the above conclusions were nevertheless only valid for the analyses based on the unbiased estimator that accounts for the additional level of variation introduced by the sampling of the DNA of pooled individuals (non identifiable) at the sequencing step. We found that improperly analyzing Pool-Seq read counts as standard allele counts had high detrimental consequence on the estimation of all the $F$ parameters that involved $Q_{1}$ probabilities (within population probability of identity) in their definition, i.e., $F_{2}, F_{S T}$ (as previously observed by Hivert et al, 2018, see also Figure S5), $F_{3}$ and $F_{3}^{\star}$ leading to a complete loss of power of the associated threepopulation test in our simulation. When processing admixture graph fitting, this also resulted in a strong upward bias in the estimation of branch lengths, including the external ones that were accurately estimated when relying on unbiased estimators. Loosely speaking, not accounting for the extra-variance introduced by the sampling of reads has the same effect of adding a (substantial) amount of extra drift explaining the two aforementioned observations. Although not investigated here (and of little interest since we should definitely rely on unbiased estimators), the amount of extra variance may be inversely proportional to the pool haploid sample size (i.e., bias may decrease with increasing pool sample size). Conversely, analyzing Pool-Seq read counts as standard allele counts did not affect the performance of the $f_{4^{-}}$(and $D$ ) based test of treeness or the estimation of admixture proportion from $F_{4^{-}}$ ratio. This was expected from the properties of the underlying parameters that only depends on the $Q_{2}$ probabilities across the different pairs of population involved in the quadruplet of interest (eq. 3) resulting in the same estimators 
(see eqns. 4 and 5) for both allele count and Pool-Seq data. More generally, analyzing Pool-Seq read count data with popular programs that were developed for standard allele count data such as those from the AdmixTools (Patterson et al, 2012) or TreeMix (Pickrell \& Pritchard, 2012) suites should definitely be avoided and, if not, results should be carefully interpreted.

\subsection{Insights into the history of the invasive species $D$. suzukii from Pool-Seq data analysis}

To illustrate both the power and limitations of $f$-statistics based methods for historical and demographic inference as implemented in poolfstat, we analyzed Pool-Seq data available for 14 populations of the invasive species D. suzukii (Olazcuaga et al, 2020). These population samples were representative of both the Asian native area and the recently invaded American area. Most of them consisted of individuals originating from the same sites as those genotyped in Fraimout et al (2017) at microsatellite markers and analyzed under an ABC-RF framework. The results remained consistent between the two studies, both of them pointing to complex invasion pathways including multiple introductions leading to admixed origins of the continental American populations. The main source contributions were from Hawaii, where D. suzukii was described about 30 years earlier and the native area (China and Japan). However, some inferred scenarios appeared somewhat conflicting. First, for the Hawaiian population that played a key role in American invasion route, both poolfstat and Fraimout et al (2017) suggested a Japanese origin. However, we here found that the sample the closest to the source (internal node population JP in Figure 2) was JP-Sap (sampled in Sapporo) while Fraimout et al (2017) rather concluded it was JP-Tok (sampled in Tokyo) which was not found to be directly contributing to US-Haw in poolfstat analyses and was even found to be admixed by native populations from Japan and China. In the ABC-RF treatments by Fraimout et al (2017), all populations from the native area were assumed to be non-admixed and no "ghost" (i.e., unsampled) populations were included in the model whereas such populations are present in admixture graphs through internal nodes. Moreover, the samples from Hawaii and Tokyo both differed in their exact location and collection date (2013 and 2016 for Hawaii, 2014 and 2016 for Tokyo) between the two studies, which may further explain the observed discrepancies and more generally promotes the sequencing of additional samples in this area to better resolve the origin of the Hawaiian population(s).

Interestingly, poolfstat results challenged the initial view about the pioneering origin of the Californian population US-Wat in the invasion of continental America (as suggested by historical records) suggesting it rather originates from an admixture between two already established but unsampled continental American populations, one presumably Northern (related to Am1 and here represented by US-Sok) and the other presumably more South- 
ern (related to Am2 and here represented by BR-Pal from Brazil and US-Sdi from South-California). This discrepancy between Fraimout et al (2017) and poolfstat results points to three key issues. First, a too strong reliance on the reported date of first observation of the species in the invasive area when formalizing the scenario to be compared in $\mathrm{ABC}$ modeling may actually mislead inference and this especially since $D$. suzukii was first observed at very close dates in the US-Wat, US-Sdi and US-Sok sampled locations (i.e., 2008, 2009 and 2009, respectively). As a matter of fact, Fraimout et al (2017) only considered scenarios in which US-Wat was the first population introduced in continental America. Second, in ABC, scenarios are defined by hand justifying the use of dates of first observation to minimize their number (Estoup \& Guillemaud, 2010). The functions implemented in poolfstat circumvent this constraint by facilitating a quick and automatic exploration of the admixture graph space to identify key historical events relating the populations of interest. Third, our finding reinforces the concern that the formalization of invasion scenarios including the possibility of unsampled populations is crucial. This possibility is by construction included in admixture graph construction but is also possible in ABC modeling (e.g. Guillemaud et al, 2010). Similarly, Fraimout et al (2017) argued for an admixed origin of the Brazilian BR-Pal population (first observed in 2013) between undefined North-Western and North-Eastern American sources, while we here found that this population was the best proxy for the ancestral "ghost" American population Am2 (Figure 2C) which may be viewed as one of the main contributor of all the sampled North-American populations (but US-Sok). Again, this results underline advantages of not relying on historical dates as for poolfstat analyses, and promotes the sequencing of additional samples in South and North-Western America areas to more thoroughly decipher the invasion routes followed by continental American populations.

If Pool-Seq data analyzed with poolfstat allowed to refine historical and demographic scenarios in both the native and invasive areas, the $D$. suzukii Pool-Seq data analysis also illustrated some inherent constraints imposed to the modeled demographic history when fitting admixture graph. In particular, more complex histories involving recurrent admixture events turned out to be difficult or even impossible to fit unless a number of source key samples are included, as observed here for the North-Eastern American populations. In real-life applications involving a large number of invasive populations characterized by numerous and recurrent introduction events, summarizing precisely and with a good fit the history of all surveyed populations with a comprehensive admixture graph may remain elusive. However, as previously underlined (Patterson et al, 2012; Lipson \& Reich, 2017; Lipson, 2020), in addition to providing robust formal tests of admixture or treeness, a decisive advantage of $f$-based inference methods is to allow straightforward assessment of the fitted admixture graph by carefully inspecting and reporting Z-score of the residuals of the fitted statistics, an option not available in other related methods such as TreeMix 
(Pickrell \& Pritchard, 2012). Beyond modeling the history of populations as admixture graphs (via formal tests of admixture of treeness or graph fitting), Peter (2016) provided valuable theoretical insights to interpret the estimated $f$-statistics under alternative demographic models such as island, stepping-stone or serial founder models. This suggests in turn that these statistics should be informative to estimate the parameters of demographic scenarios more complex than admixture graphs (e.g., under an ABC framework as in Collin et al, 2021).

\section{Acknowledgements}

This work was supported by the French National Research Agency (ANR) for the projects SWING (ANR-16CE02-0015-01) and GANDHI (ANR-20-CE02-0018).

\section{Data Accessibility}

The vcf file generated for the $D$. suzukii Pool Seq data is publicly available from the Zenodo repository (http: //doi.org/10.5281/zenodo.4709080). Further details are provided in Supplementary Vignettes V1 and V2.

\section{Supporting Information}

Additional supporting information is available online and consists of three pdf files: i) vignette V1 for the R package poolfstat providing a detailed tutorial using Pool-Seq and allele count data simulated under the scenario depicted in Figure 1; ii) vignette V2 detailing the analysis of the real Drosophila suzukii Pool-Seq data using the R package poolfstat; and iii) a single pdf file with the sixteen supplementary Tables and the five supplementary Figures. 


\section{References}

Adrion JR, Kousathanas A, Pascual M, et al (2014) Drosophila suzukii: The genetic footprint of a recent, worldwide invasion. Molecular Biology and Evolution, 31, 3148-3163.

Andersen MM, Højsgaard S (2019) Ryacas: A computer algebra system in R. Journal of Open Source Software, 4.

Bhatia G, Patterson N, Sankararaman S, Price AL (2013) Estimating and interpreting Fst: the impact of rare variants. Genome Research, 9, 1514-21.

Busing FMTA, Meijer E, Leeden RVD (1999) Delete-m jackknife for unequal m. Statistics and Computing, 9 , $3-8$.

Collin FD, Durif G, Raynal L, et al (2021) Extending approximate bayesian computation with supervised machine learning to infer demographic history from genetic polymorphisms using diyabc random forest. Molecular Ecology Resources, accepted.

Durand EY, Patterson N, Reich D, Slatkin M (2011) Testing for ancient admixture between closely related populations. Molecular biology and evolution, 28, 2239-2252.

Eddelbuettel D (2013) Seamless $R$ and $C++$ Integration with Rcpp. Springer, New York.

Estoup A, Guillemaud T (2010) Reconstructing routes of invasion using genetic data: why, how and so what? Molecular ecology, 19, 4113-4130.

Feder AF, Petrov DA, Bergland AO (2012) LDx: estimation of linkage disequilibrium from high-throughput pooled resequencing data. PLoS One, 7, e48588.

Ferretti L, Ramos-Onsins SE, Pérez-Enciso M (2013) Population genomics from pool sequencing. Molecular Ecology, 22, 5561-5576.

Fraimout A, Debat V, Fellous S, et al (2017) Deciphering the routes of invasion of drosophila suzukii by means of abc random forest. Molecular biology and evolution, 34, 980-996.

Garrison E, Marth G (2012) Haplotype-based variant detection from short-read sequencing. arXiv, p. 1207.3907. 
Gautier M (2015) Genome-wide scan for adaptive divergence and association with population-specific covariates. Genetics, 201, 1555-1579.

Gautier M, Foucaud J, Gharbi K, et al (2013) Estimation of population allele frequencies from next-generation sequencing data: pool-versus individual-based genotyping. Molecular Ecology, 22, 3766-3779.

Glenn TC (2011) Field guide to next-generation dna sequencers. Molecular Ecology Resources, 11, 759-769.

Green RE, Krause J, Briggs AW, et al (2010) A draft sequence of the neandertal genome. Science (New York, N.Y.), 328, 710-722.

Guillemaud T, Beaumont MA, Ciosi M, Cornuet JM, Estoup A (2010) Inferring introduction routes of invasive species using approximate bayesian computation on microsatellite data. Heredity, 104, 88-99.

Hivert V, Leblois R, Petit EJ, Gautier M, Vitalis R (2018) Measuring genetic differentiation from pool-seq data. Genetics, 210, 315-330.

Iannone R (2020) DiagrammeR: Graph/Network Visualization. R package version 1.0.6.1.

Kass RE, Raftery AE (1995) Bayes factors. Journal of the American Statistical Association, 90, 773-795.

Kelleher J, Etheridge AM, McVean G (2016) Efficient coalescent simulation and genealogical analysis for large sample sizes. PLoS Computational Biology, 12, e1004842.

Knaus BJ, Grünwald NJ (2017) vcfr: a package to manipulate and visualize variant call format data in R. Molecular Ecology Resources, 17, 44-53.

Koboldt DC, Zhang Q, Larson DE, et al (2012) Varscan 2: somatic mutation and copy number alteration discovery in cancer by exome sequencing. Genome Research, 22, 568-576.

Kofler R, Orozco-terWengel P, De Maio N, et al (2011) Popoolation: a toolbox for population genetic analysis of next generation sequencing data from pooled individuals. PloS One, 6, e15925.

Kunsch HR (1989) The jackknife and the bootstrap for general stationary observations. Annals of Statistics, 17, $1217-1241$.

Lawson CL, Hanson RJ (1995) Solving least squares problems. No. 15 in Classics in applied mathematics. Society for industrial and applied mathematics, 1995, 1st edn.. 
Leblois R, Gautier M, Rohfritsch A, et al (2018) Deciphering the demographic history of allochronic differentiation in the pine processionary moth thaumetopoea pityocampa. Molecular Ecology, 27, 264-278.

Leppälä K, Nielsen SV, Mailund T (2017) admixturegraph: an r package for admixture graph manipulation and fitting. Bioinformatics, 33, 1738-1740.

Li H, Handsaker B, Wysoker A, et al (2009) The sequence alignment/map format and SAMtools. Bioinformatics, 25, 2078-2079.

Lipson M (2020) Applying f-statistics and admixture graphs: Theory and examples. Molecular Ecology Resources, 20, 1658-1667.

Lipson M, Loh PR, Levin A, Reich D, Patterson N, Berger B (2013) Efficient moment-based inference of admixture parameters and sources of gene flow. Molecular Biology and Evolution, 30, 1788-1802.

Lipson M, Loh PR, Patterson N, et al (2014) Reconstructing austronesian population history in island southeast asia. Nature Communications, 5, 4689.

Lipson M, Reich D (2017) A working model of the deep relationships of diverse modern human genetic lineages outside of africa. Molecular Biology and Evolution, 34, 889-902.

Long Q, Jeffares DC, Zhang Q, et al (2011) PoolHap: inferring haplotype frequencies from pooled samples by next generation sequencing. PLoS One, 6, e15292.

McKenna A, Hanna M, Banks E, et al (2010) The genome analysis toolkit: a mapreduce framework for analyzing next-generation dna sequencing data. Genome Research, 20, 1297-1303.

Nocedal J, Wright SJ (1999) Numerical optimization. Springer series in operations research. Springer, New York, NY [u.a.].

Olazcuaga L, Loiseau A, Parrinello H, et al (2020) A whole-genome scan for association with invasion success in the fruit fly drosophila suzukii using contrasts of allele frequencies corrected for population structure. Molecular biology and evolution, 37, 2369-2385.

Paradis E, Claude J, Strimmer K (2004) Ape: Analyses of phylogenetics and evolution in r language. Bioinformatics, 20, 289-290. 
Paris M, Boyer R, Jaenichen R, et al (2020) Near-chromosome level genome assembly of the fruit pest drosophila suzukii using long-read sequencing. Scientific reports, 10, 11227.

Patterson N, Moorjani P, Luo Y, et al (2012) Ancient admixture in human history. Genetics, 192, 1065-1093.

Peter BM (2016) Admixture, population structure, and f-statistics. Genetics, 202, 1485-1501.

Petr M, Vernot B, Kelso J (2019) admixr-r package for reproducible analyses using admixtools. Bioinformatics, 35, 3194-3195.

Pickrell JK, Pritchard JK (2012) Inference of population splits and mixtures from genome-wide allele frequency data. PLoS Genetics, 8, e1002967.

Reich D, Thangaraj K, Patterson N, Price AL, Singh L (2009) Reconstructing indian population history. Nature, 461, 489-494.

Rousset F (2007) Inferences from spatial population genetics. In Handbook of Statistical Genetics (edited by DJ Balding, M Bishop, C Cannings), pp. 945-979. John Wiley and Sons, Ltd, Chichester, England, 3rd edn..

Schlötterer C, Tobler R, Kofler R, Nolte V (2014) Sequencing pools of individuals - mining genome-wide polymorphism data without big funding. Nature Reviews Genetics, 15, 749-763.

Slatkin M (1991) Inbreeding coefficients and coalescence times. Genetical research, 58, 167-175.

Weir BS (1996) Genetic data analysis II : methods for discrete population genetic data. Sinauer Associates, Sunderland, Mass.

Weir BS, Goudet J (2017) A unified characterization of population structure and relatedness. Genetics, 206, 20852103. 